\title{
ON VARIETIES OF METABELIAN GROUPS OF PRIME-POWER EXPONENT
}

\author{
M. S. BROOKS \\ (Received 1 July 1970) \\ Communicated by G. E. Wall
}

Let $\mathfrak{A}_{n}$ denote the variety of abelian groups of exponent dividing $n$, and let $p$ be an arbitrary prime. In this paper all non-nilpotent, join-ireducible subvarieties of the product variety $\mathfrak{A}_{p} \mathfrak{A}_{p^{2}}$ are determined. The proper subvarieties of this kind in fact form an infinite ascending chain $\mathfrak{I}_{1} \subset \mathfrak{I}_{2} \subset \cdots$, and an arbitrary proper subvariety $\mathfrak{V}$ of $\mathfrak{U}_{p} \mathfrak{A}_{p^{2}}$ is either nilpotent or a join $\mathfrak{I}_{k} \vee \mathfrak{L}$, where $\mathfrak{L}$ is nilpotent and $k$ is uniquely determined by $\mathfrak{B}$.

\section{Introduction}

Notation and terminology generally follows Neumann's book [11]; in particular $\mathfrak{A}_{0}$ or $\mathfrak{A}$ is used to denote the variety of all abelian groups and $\mathfrak{B}_{n}, \mathfrak{A}_{n}$ and $\mathfrak{N}_{c}$ denote respectively the varieties of all groups of exponent dividing $n$, all abelian groups of exponent dividing $n$, and all nilpotent groups of class at most $c$. Unless otherwise stated, $p$ denotes an arbitrary prime.

If $\mathfrak{B}$ is any variety, the set of all subvarieties of $\mathfrak{B}$ forms a lattice with respect to the inclusion ordering. This lattice of subvarieties will be denoted by lat $(\mathfrak{B})$, "Determining" lat $(\mathfrak{B})$ shall mean determining all subvarieties of $\mathfrak{B}$ and describing the inclusion ordering on them.

The work reported here relates to the general problem of determining lat( $\mathfrak{H} \mathfrak{H})$. By Cohen [4] this lattice has minimum condition, and so every metabelian variety $\mathfrak{W}$ can be expressed as the irredundant join of finitely many join-irreducible subvarieties. Since the lattice is not distributive (Kovács and Newman (unpublished) - see also [2]), not every $\mathfrak{M}$ has a unique expression of this kind, but nevertheless a classification of all join-irreducible metabelian varieties would clearly provide a great deal of information about lat( $\mathfrak{H} \mathfrak{H})$. In this direction Kovács and Newman [6] have already classified the infinite exponent ones, and those of finite composite exponent have been considered by Bryce, who has obtained a powerful reduction theorem ([3], 6.1.8 and 4.2.33(i)). The prime-power exponent case has also been attacked, notably by Brisley [1] and Weichsel [13], who have determined 
lat $\left(\mathfrak{A} \mathfrak{A} \wedge \mathfrak{B}_{p^{a}} \wedge \mathfrak{N}_{p-1}\right)$ for all $a^{1}$, and by Kovács and Newman [7] who have determined lat $\left(\mathfrak{A}_{p^{a}} \mathfrak{A}_{p}\right)$ for all $a$. (In fact in [6] Kovács and Newman determine lat $\left(\mathfrak{A P}_{n}\right)$ for all square-free $n$.) This paper is concerned with another primepower exponent case, namely $\mathfrak{U}_{p} \mathfrak{A}_{p^{2}}$, but unfortunately a complete determination of all subvarieties, or even of all join-irreducible ones, is not achieved here. Part of the difficulty is that, in contrast to lat $\left(\mathfrak{U} \mathfrak{A} \wedge \mathfrak{B}_{p^{a}} \wedge \mathfrak{N}_{p-1}\right)$ and lat $\left(\mathfrak{H}_{p^{a}} \mathfrak{A}_{p}\right)$, lat $\left(\mathfrak{U}_{p} \mathfrak{H}_{p^{2}}\right)$ is not distributive (see [2]). Moreover, again in contrast to the other two cases mentioned, $\mathfrak{A}_{p} \mathfrak{A}_{p^{2}}$ contains infinitely many non-nilpotent join-irreducible subvarieties (see 1.2 below).

What will be proved is the following:

1.1 THFOREM. The varieties $\mathfrak{I}_{k}, k=1,2, \cdots$, defined by

$$
\mathfrak{I}_{k}=\left\{\begin{array}{ll}
\mathfrak{A}_{p} \mathfrak{H}_{p^{2}} \wedge \mathfrak{R}_{k} \mathfrak{A}_{p} \wedge \mathfrak{B}_{p^{2}}, & \text { if } 1 \leqq k \leqq p-1 \\
\mathfrak{A}_{p} \mathfrak{A}_{p^{2}} \wedge \mathfrak{N}_{k} \mathfrak{A}_{p}, & \text { if } \quad p \leqq k
\end{array}\right\}
$$

have the following properties:

a) A proper subvariety $\mathfrak{M}$ of $\mathfrak{A}_{p^{2}} \mathfrak{A}_{p^{2}}$ is non-nilpotent if, and only if, $\mathfrak{W}=\mathfrak{I}_{k} \vee \mathfrak{L}$ for some $k \in\{1,2, \cdots\}$ and some nilpotent variety $\mathfrak{Q}$.

b) If $\mathfrak{L}$ and $\mathfrak{Q}^{*}$ are nilpotent subvarieties of $\mathfrak{A}_{p} \mathfrak{A}_{p^{2}}$ and $\mathfrak{I}_{k} \vee \mathfrak{L}=\mathfrak{I}_{k^{*}} \vee \mathfrak{L}^{*}$, then $k=k^{*}$.

There is an immediate corollary.

1.2 COROLlaRY. The $\mathfrak{I}_{k}$ form a properly ascending chain of subvarieties of $\mathfrak{A}_{p} \mathfrak{H}_{p^{2}}$, and this chain, with $\mathfrak{H}_{p} \mathfrak{H}_{p^{2}}$ itself adjoined, makes up a complete list of the non-nilpotent join-irreducible subvarieties of $\mathfrak{A}_{p} \mathfrak{H}_{p^{2}}$.

Some remarks on the theorem follow:

1) The theorem says that an arbitrary non-nilpotent proper subvariety $\mathfrak{B}$ of $\mathfrak{A}_{p} \mathfrak{H}_{p^{2}}$ can be expressed in the form $\mathfrak{W}=\mathfrak{I}_{k} \vee \mathfrak{L}$, where $\mathfrak{L}$ is nilpotent and $k$ is uniquely determined by $\mathfrak{W}$. Clearly, however, $\mathfrak{L}$ is not uniquely determined by $\mathfrak{W}$; for example, it can always be enlarged by adjoining a nilpotent subvariety of $\mathfrak{B}$ with sufficiently high class. Nevertheless, since lat $(\mathfrak{L})$ has minimum condition (by Lyndon [9]), there does exist a nilpotent variety, $\mathfrak{L}_{\text {min }}$ say, such that $\mathfrak{W}=\mathfrak{I}_{k} \vee \mathfrak{L}_{\text {min }}$ but $\mathfrak{B} \neq \mathfrak{I}_{k} \vee \mathfrak{L}_{1}$ for any $\mathfrak{L}_{1}<\mathfrak{L}_{\text {min }}$. The question naturally arises as to whether such a "minimal nilpotent component" is uniquely determined by $\mathfrak{M}$. The answer is no: it was shown in [2] that, for $p=3$ at least, there exists a subvariety $\mathfrak{M}$ of $\mathfrak{A}_{p} \mathfrak{H}_{p^{2}}$ such that $\mathfrak{B}=\mathfrak{I}_{p} \vee \mathfrak{L}=\mathfrak{I}_{p} \vee \mathfrak{L}^{*}$, where $\mathfrak{L}$ and $\mathfrak{L}^{*}$ are distinct nilpotent varieties each minimal with respect to the property that its join with $\mathfrak{I}_{p}$ is $\mathfrak{W}$.

1 Brisley can now deal with class $p+1$; see his "Varieties of metabelian $p$-groups of class $p, p+1 "$ in this Journal, 12, 53-62. 
2) Minimum condition (which lat $\left(\mathfrak{H}_{p} \mathfrak{A}_{p^{2}}\right)$ satisfies by Cohen) is not used in the proof of 1.1, and in fact the converse situation obtains: minimum condition on lat $\left(\mathfrak{H}_{p} \mathfrak{H}_{p^{2}}\right)$ is a consequence of 1.1 by virtue of Lyndon's theorem mentioned above and the fact that minimum condition is always satisfied by a modular lattice in which every element is a join of finitely many join-irreducibles and the set of join irreducibles has minimum condition. This last fact was proved by Kovács in [5] for join-continuous modular lattices, and just recently a much simpler proof, which does not use join-continuity, has been given by Newman [12].

3) Theorem 1.1 shows that the non-nilpotent join-irreducible subvarieties of $\mathfrak{U}_{p^{2}} \mathfrak{H}_{p^{2}}$ can be obtained in a rather simple manner from certain nilpotent joinirreducible varieties of smaller exponent, for it may be easily verified that $\mathfrak{I}_{k}=\overline{\mathfrak{J}}_{k} \mathfrak{A}_{p} \wedge \mathfrak{A}_{p} \mathfrak{A}_{p^{2}}$ for all $k$, where the varieties $\overline{\mathfrak{I}}_{k}$ are defined by

$$
\overline{\mathfrak{I}}_{k}=\left\{\begin{array}{ll}
\mathfrak{A}_{p} \mathfrak{A}_{p} \wedge \mathfrak{N}_{k} \wedge \mathfrak{B}_{p}, & \text { if } 1 \leqq k \leqq p-1 \\
\mathfrak{A}_{p} \mathfrak{A}_{p} \wedge \mathfrak{N}_{k}, & \text { if } p \leqq k
\end{array}\right\},
$$

and are all join-irreducible by Kovács and Newman[7]. It would be interesting to know what statements along these lines can be made about non-nilpotent join-irreducible subvarieties of $\mathfrak{A}_{p} \mathfrak{A}_{p^{\beta}}$ for arbitrary $\beta$, especially in view of Bryce's Theorem 4.2 .33 in [3].

The bones of a proof of 1.1 are given in $\S 4$. Sections 2 and 3 are preparatory for this and sections 5 to 10 fill in the details.

This paper constitutes a revised and abbreviated version of the principal part of the author's Ph.D. thesis (Australian National, University 1968). I gratefully acknowledge both the help given me by my supervisors, Dr. L. G. Kovács and Dr. M. F. Newman, and the financial support of the University.

\section{Preliminaries: commutator calculus}

Standard notation will be used for left-normed commutators: If $h_{1}, h_{2}, \cdots$ are elements of a group $H$, then

(i) $\left[h_{1}, h_{2}\right]=h_{1}^{-1} h_{2}^{-1} h_{1} h_{2}$;

(ii) for $k>2,\left[h_{1}, h_{2}, \cdots, h_{k}\right]=\left[\left[h_{1}, \cdots, h_{k-1}\right], h_{k}\right]$;

(iii) $\left[h_{1}, 0 h_{2}\right]=h_{1}$ and $\left[h_{1}, r h_{2}\right]=\left[\left[h_{1},(r-1) h_{2}\right], h_{2}\right]$ for $r>0$; and

(iv) for non-negative integers $r_{2}, \cdots, r_{k}$ with $k>2$,

$$
\left[h_{1}, r_{2} h_{2}, \cdots, r_{k} h_{k}\right]=\left[\left[h_{1}, r_{2} h_{2}, \cdots, r_{k-1} h_{k-1}\right], r_{k} h_{k}\right] .
$$

If $H_{1}, H_{2}$ are subgroups of a group $H$ then $\left[H_{1}, H_{2}\right]=\operatorname{gp}\left(\left[h_{1}, h_{2}\right] \mid h_{i} \in H_{i}\right)$ and for $r \geqq 0\left[H_{1}, r H_{2}\right]$ is defined recursively as (iii) above. The $c$ th term of the lower central series of a group $H$ will usually be denoted by $H_{(c)}$; that is $H_{(c)}=[H,(c-1) H]$. 
The proof of Theorem 1.1 consists largely of a detailed investigation of two parameters, called "weight" and " $p$-complexity" (see $\S 4$ ), which will be assigned to elements, that is products of commutators, in the abelian derived group of $G=F_{\infty}\left(\mathfrak{A}_{p} \mathfrak{A}_{p^{2}}\right)$. These parameters in fact belong more properly to the manner in which the elements are expressed, so in order to avoid ambiguity it is necessary to construct a one-to-one correspondence between elements of $G^{\prime}$ and words in the language used to express them. This is accomplished firstly by defining "pseudocommutators" in 2.2 below, and secondly by obtaining, as a special case of Theorem 3.1, a basis for $G^{\prime}$. The following well-known result provides some motivation for Definition 2.2:

2.1 LeMMA (34.51 in [11]). If $H$ is a metabelian group and $h_{1}, \cdots, h_{k} \in H$ with $k \geqq 2$, then

$$
\left[h_{1}, h_{2}, h_{3}, \cdots, h_{k}\right]=\left[h_{1}, h_{2}, h_{3 \pi}, \cdots, h_{k \pi}\right]
$$

for every permutation $\pi$ of $\{3, \cdots, k\}$.

2.2 Definition. Let $H$ be a metabelian group. A degree function on $H$ is a function $\delta: H \rightarrow\{0,1,2, \cdots\}$ whose support

$$
\operatorname{supp} \delta=\{h \in H \mid \delta(h) \neq 0\}
$$

is a finite but non-empty set. A pseudo-commutator in $H$ is an ordered triple $\tilde{p}=\left(h_{1}, h_{2}, \delta\right)$ in which $\delta$ is a degree function on $H$ and $h_{1}, h_{2} \in \operatorname{supp} \delta$. The pseudo-commutator $\tilde{p}$ will be called trivial if $h_{1}=h_{2}$. For any $h \in H$, the integer $\delta(h)$ will be called the degree of $h$ in $\tilde{p}$. If $\delta(h) \neq 0$ then $h$ will be called an entry in $\tilde{p}$, and the weight of $\tilde{p}$ is defined to be the sum of the degrees of its entries. Thus the set of entries in $\tilde{p}$ is $\operatorname{supp} \delta$, and the weight of $\tilde{p}$, which will be denoted by $w t(\tilde{p})$, is given by

$$
\operatorname{wt}(\tilde{p})=\sum_{h \in \operatorname{supp} \delta} \delta(h)=\sum_{\in H} \delta(h) .
$$

If $\operatorname{supp} \delta=\left\{h_{1}, \cdots, h_{k}\right\}$ then the element

$$
\left[h_{1}, \delta\left(h_{2}\right) h_{2}, \delta\left(h_{3}\right) h_{3}, \cdots, \delta\left(h_{k}\right) h_{k},\left(\delta\left(h_{1}\right)-1\right) h_{1}\right]
$$

will be called the value of $\tilde{p}=\left(h_{1}, h_{2}, \delta\right)$ and will be denoted by $[\tilde{p}]=\left[h_{1}, h_{2}, \delta\right]$. (Lemma 2.1 ensures that $[\tilde{p}]$ is well-defined.) The set of all pseudo-commutators in $H$ will be denoted by $\tilde{P}(H)$.

The following well-known and easily verifiable commutator expansion rules for an arbitrary metabelian group $H$ will frequently be used without explicit mention:

(i) If $a, b, c \in H$, then 


$$
[a b, c]=[a, c][b, c][a, c, b]
$$

and

$$
[a, b c]=[a, b][a, c][a, b, c] .
$$

(ii) If $h_{1}, h_{2}, \cdots, \in H$ and $c_{1}, c_{2}, \cdots, \in H^{\prime}$, then

$$
\left[\prod_{i} c_{i}, h_{1}, h_{2}, \cdots\right]=\prod_{i}\left[c_{i}, h_{1}, h_{2}, \cdots\right] .
$$

(iii) If $a, b \in H$, then, for any $k \geqq 1$,

and

$$
\left[a^{k}, b\right]=\prod_{c=1}^{k}[a, b,(i-1) a]^{\left(\begin{array}{c}
k \\
i
\end{array}\right)}
$$

$$
\left[a, b^{k}\right]=\prod_{i=1}^{k}[a, i b]^{\left(\begin{array}{l}
k \\
i
\end{array}\right)} .
$$

(iv) (The Jacobi identity) If $\delta$ is a degree function on $H$ and $h_{1}, \cdots, h_{k} \in \operatorname{supp} \delta$ for $k \geqq 1$, then

$$
\left[h_{k}, h_{1}, \delta\right]\left[h_{1}, h_{2}, \delta\right]\left[h_{2}, h_{3}, \delta\right] \cdots\left[h_{k-1}, h_{k}, \delta\right]=1 .
$$

Using the laws $\left[x^{n}, y^{n}\right]$ and $\left[x, y, z^{n}\right]$ together with the above expansion rules it is a routine matter to establish the following lemma, which is needed for the proof of Theorem 3.1:

2.3 Lemma. Let $H \in \mathfrak{A}_{m} \mathfrak{P}_{n}(n \neq 0)$ and let $a, b, c \in H$.

a)

$$
\left[a, b^{-1}\right]=\prod_{i=1}^{n}[a, i b]^{-\left(\begin{array}{c}
n-1 \\
i-1
\end{array}\right)} \text {. }
$$

b) For any positive integer $k$ there exist integers $e_{0}(k), \cdots, e_{n-1}(k)$ such that

$$
[a, b, k c]=\prod_{i=0}^{n-1}[a, b, i c]^{e_{i}(k)} .
$$

c) If $a \neq b, \delta^{*}$ is a degree function on $H$ and, for $i, j=1, \cdots, n, \delta_{i j}$ is the degree function defined by $\delta_{i j}(a)=i, \delta_{i j}(b)=j$ and $\delta_{i j}(d)=\delta^{*}(d)$ for all $d \in H \backslash\{a, b\}$, then

$$
\left[a, b, \delta_{n n}\right]=\prod_{\substack{i=1 \\
i+j}}^{n} \prod_{\substack{j=1 \\
<2 n}}^{n}\left[a, b, \delta_{i j}\right]^{-\left(\begin{array}{l}
n \\
i
\end{array}\right)\left(\begin{array}{l}
n \\
j
\end{array}\right)} .
$$

d) If $\delta^{*}$ is a degree function on $H$ with $a, c \in \operatorname{supp} \delta$, and if for $i=1, \cdots, n$ the degree function $\delta_{i}$ is defined by $\delta_{i}(b)=i$ and $\delta_{i}(d)=\delta^{*}(d)$ for all $d \in H \backslash\{b\}$, then

$$
\left[a, b, \delta_{n}\right]=\left[c, b, \delta_{n}\right] \prod_{i=1}^{n-1}\left(\left[c, b, \delta_{i}\right]\left[a, b, \delta_{i}\right]^{-1}\right)^{\left(\begin{array}{l}
n \\
i
\end{array}\right)}
$$




\section{The derived group of $F_{\infty}\left(\mathfrak{A}_{m} \mathfrak{A}_{n}\right)$}

In this section the structure of the derived group $F_{\infty}^{\prime}\left(\mathfrak{A}_{m} \mathfrak{A}_{n}\right)$ of $F_{\infty}\left(\mathfrak{A}_{m} \mathfrak{A}_{n}\right)$ is investigated. Since $\mathfrak{A}_{1}$ is the variety of trivial groups, $F_{\infty}^{\prime}\left(\mathfrak{A}_{m} \mathfrak{A}_{n}\right)$ is trivial if $m$ or $n$ is 1 . On the other hand, for $n=0$ the structure of $F_{\infty}^{\prime}\left(\mathfrak{A}_{m} \mathfrak{A}_{n}\right)$ is more complicated than can be handled by the methods presented here. Consequently, throughout this section let $m$ and $n$ be arbitrary but fixed non-negative integers with $m \neq 1, n>1$, and let $G=F_{\infty}\left(\mathfrak{U}_{m} \mathfrak{A}_{n}\right)$. Although Theorem 3.1 below is needed only for the case $m=p, n=p^{2}$, it is given for general $m, n$ as this does not make the proof any more difficult.

Let $\mathfrak{g}$ be a fixed ordered free generating set for $G$, say $\mathfrak{g}=\left\{g_{1}, g_{2}, \cdots\right\}$ with $g_{i} \leqq g_{j}$ if and only if $i \leqq j$. The aim is to prove the following:

3.1 THEOREM. a) The derived group $G^{\prime}$ of $G$ is free abelian of exponent $m$.

b) If $\widetilde{B}$ is the set of those non-trivial pseudo-commutators $\tilde{p}=(a, b, \delta)$ in $G$ which satisfy the five conditions below, then the valuation mapping $\tilde{p} \mapsto[\tilde{p}]$ of $\widetilde{B}$ into $G$ is one-to-one, and its image $B$ is a basis for $G^{\prime}$.

Condition (1): $\operatorname{supp} \delta \subseteq \mathfrak{g}$.

" (2): $\delta(c)<n$ for all $c \in \mathfrak{g} \backslash\{a, b\}$.

, (3): $\delta(a) \leqq n, \delta(b) \leqq n$ and $\delta(a)+\delta(b)<2 n$.

" (4): $b=\min \operatorname{supp} \delta$ (i.e., $b$ is the least element in $\operatorname{supp} \delta$ ).

, (5): If $\delta(b)=n$, then $a=\max \operatorname{supp} \delta$.

For each positive integer $r$, let $G_{r}$ be the subgroup of $G$ generated by $\mathfrak{g}_{r}=\left\{g_{1}, \cdots, g_{r}\right\} \subseteq \mathfrak{g}$ (so that $G_{r} \cong F_{r}\left(\mathfrak{A}_{m} \mathfrak{A}_{n}\right)$ ). Part a) of 3.1 is an immediate consequence of

3.2 Lemma. If $r \geqq 2$, then $G_{r}{ }^{\prime}$ is free abelian of exponent $m$ : its rank is $(r-1)\left(n^{r}-1\right)$.

Proof. Let $F_{r}$ be an absolutely free group of rank $r$. Since $F_{r} / A_{n}\left(F_{r}\right) \cong F_{r}\left(\mathfrak{A}_{n}\right)$, it follows using Schreier's formula that

$$
A_{n}\left(F_{r}\right) / A_{m}\left(A_{n}\left(F_{r}\right)\right) \cong F_{(r-1) n^{r}+1}\left(\mathfrak{U}_{m}\right) .
$$

Also, it is clear that $A_{n}\left(F_{r}\left(\mathfrak{A}_{m n}\right) \cong F_{r}\left(\mathfrak{A}_{m}\right)\right.$, which means that

$$
A_{n}\left(F_{r}\right) / A_{m n}\left(F_{r}\right) \cong F_{r}\left(\mathfrak{A}_{m}\right) \text {. }
$$

From these two isomorphisms it follows that

$$
A_{m n}\left(F_{r}\right) / A_{m}\left(A_{n}\left(F_{r}\right)\right) \cong F_{(r-1) n^{r+1-r}}\left(\mathfrak{A}_{m}\right)=F_{(r-1)\left(n^{r}-1\right)}\left(\mathfrak{A}_{m}\right),
$$

which is what the lemma asserts.

Now set $\widetilde{B}_{r}=\widetilde{B} \cap \tilde{P}\left(G_{r}\right)$. For the proof of $\left.3.1 \mathrm{~b}\right)$ it is clearly sufficient to show that, for all $r \geqq 2$, the valuation mapping $\tilde{p} \mapsto[\tilde{p}]$ of $\widetilde{B}_{r}$ into $G_{r}$ is one-to-one 
and that the image $B_{r}$ of this mapping is a basis for $G_{r}^{\prime}$. But a straightforward numerical computation shows that $\tilde{B}_{r}$ has the right order for this, namely $(r-1)\left(n^{r}-1\right)$, so it remains to prove

3.3 LEMMA. If $r \geqq 2$, then $B_{r}$ is a generating set for $G_{r}{ }^{\prime}$.

PRoof. A sketch will be sufficient:

By definition, $G_{r}^{\prime}$ is generated by $\left\{[u, v] \mid u, v \in G_{r}\right\}$. By writing $u=u_{1} \cdots u_{l(u)}$, $v=v_{1} \cdots v_{l(r)}$, where each $u_{i}, v_{j}$ is either a member of $\mathfrak{g}_{r}$ or the inverse of such, and by making multiple applications of (i) the commutator expansion rules for metabelian groups and (ii) part a) of Lemma 2.3, it can clearly be shown that $[u, v]$ can be expressed as the product of left-normed commutators all of whose entries come from $\mathfrak{g}_{r}$. Thus $\left[\widetilde{S}_{r}\right]=\left\{[\tilde{p}] \mid \tilde{p} \in \widetilde{S}_{r}\right\}$ generates $G_{r}^{\prime}$, where

$$
\tilde{S}_{r}=\left\{(a, b, \delta) \in \tilde{P}\left(G_{r}\right) \mid \operatorname{supp} \delta \subseteq \mathfrak{g}_{r}\right\} .
$$

To show that any member of $\left[\widetilde{S}_{r}\right]$ can be written as the product of elements of $\left[\widetilde{B}_{r}\right]$; and, hence that $B_{r}$ generates $G_{r}^{\prime}$, simply requires the appropriate applications of parts b), c) and d) of Lemma 2.3.

\section{A skeleton proof}

This section comprises a series of lemmas which culminate in the proof of Theorem 1.1. In the interests of simplicity of presentation the proofs of six fundamental lemmas are postponed until later sections, but apart from these the argument is complete.

From now on, $G$ always denotes the group $F_{\infty}\left(\mathfrak{A}_{p} \mathfrak{A}_{p^{2}}\right)$; that is, $m=p$ and $n=p^{2}$ throughout $\$ 4-\$ 10$. Theorem 3.1 says, then, that $G^{\prime}$ is free abelian of exponent $p$ and that $[\widetilde{B}]$ is a basis for it, where $\widetilde{B}$ is the set of basic pseudo-commutators in $G$-those pseudo-commutators $(a, b, \delta)$ in $G$ for which (1) $\operatorname{supp} \delta$ $\subseteq \mathfrak{g}$; (2) $\delta(c)<p^{2}$ for all $c \in \mathfrak{g} \mid\{a, b\}$; (3) $\delta(a) \leqq p^{2}, \delta(b) \leqq p^{2}$ and $\delta(a)+\delta(b)<2 p^{2}$; (4) $b=\min \operatorname{supp} \delta$; and (5) if $\delta(b)=p^{2}$, then $a=\max \operatorname{supp} \delta$. A non-trivial element $w$ of $G^{\prime}$ will be said to be expressed in normal form when written $w=b_{1}^{e_{1}} \cdots b_{s}^{e_{s}}$ with $b_{1}, \cdots, b_{s}$ pairwise distinct basis elements (i.e. members of $[\tilde{B}]$ ) and $e_{1}, \cdots, e_{s}$ integers not congruent to 0 modulo $p$.

A basic pseudo-commutator $\tilde{s}=(a, b, \delta)$ in $G$ with $a=g_{2}, b=g_{1}$ and $\delta\left(g_{1}\right)=\delta\left(g_{2}\right)=1$ will be termed special, and its p-complexity, comp( $\left.\tilde{s}\right)$, is defined by

$$
\operatorname{comp}(\tilde{s})=1+\sum_{i=1}^{\infty} \text { int. part }\left(\delta\left(g_{i}\right) / p\right),
$$

where int. part $\left(\delta\left(g_{i}\right) / p\right)$ is the unique integer $j_{i}$ satisfying $j_{i} \leqq \delta(g) / p<j_{i}+1$. (To see what $p$-complexity measures, read 4.1 and parts (a) and (b) of Lemma D (4.5) and then compare Lemma 4.11 with Lemma A (4.2).) 
4.1 Definition. Let $w$ be a non-trivial element of $G^{\prime}$ expressed in normal form by $w=\left[\tilde{b}_{1}\right]^{e_{1}} \cdots\left[\tilde{b}_{s}\right]_{s}^{e_{s}}$, where, of course, $\tilde{b}_{1}, \cdots, \tilde{b}_{s} \in \tilde{P}$. The weight of $w$, denoted by $\operatorname{wt}(w)$, is defined to be $\min _{j}\left(\operatorname{wt}\left(\tilde{b}_{j}\right) \mid j=1, \cdots, s\right)$. If each $\tilde{b}_{j}$ is special, then $w$ is itself termed special, and its p-complexity, denoted by $\operatorname{comp}(w)$, is defined to be $\min _{j}\left(\operatorname{comp}\left(\tilde{b}_{j}\right) \mid j=1, \cdots, s\right)$. The trivial element is also considered to be special, but both its weight and its $p$-complexity are taken as greater than that of every non-trivial element; say $\operatorname{wt}(1)=\operatorname{comp}(1)=\omega$.

Note that for $w_{1}, w_{2} \in G^{\prime}$

$$
\operatorname{wt}\left(w_{1} w_{2}\right) \geqq \min \left(\operatorname{wt}\left(w_{1}\right) \operatorname{wt}\left(w_{2}\right)\right),
$$

and that this inequality can be strict. Also, if $w_{1}$ and $w_{2}$ are both special, then so is $w_{1} w_{2}$, and

$$
\operatorname{comp}\left(w_{1} w_{2}\right) \geqq \min \left(\operatorname{comp}\left(w_{1}\right), \operatorname{comp}\left(w_{2}\right)\right),
$$

where again the inequality can be strict.

Throughout this and all subsequent sections let $G^{p^{2}}$ and $M$ denote the $\mathfrak{B}_{p^{2}}$ and $\mathfrak{H}_{p}$-subgroups of $G$ respectively; $G^{p^{2}}=B_{p^{2}}(G)$ and $M=A_{p}(G)$. (Thus $M$ is the unique maximal verbal subgroup of $G$ and also the Frattini subgroup of $G$. Note that $M_{(c)}$ is the $\mathfrak{R}_{c-1} \mathfrak{H}_{p}$-subgroup of $G$, and so in particular it is the $\mathfrak{I}_{c-1^{-}}$ subgroup of $G$ if $c>p$.) The six fundamental lemmas on which the proof of Theorem 1.1 depends are stated below. The proofs of these lemmas occupy $\$ 5$ to $\$ 10$.

4.2 LEMmA A. If $k \geqq 2$ and $w \in G_{(k)}$, then $w \mathrm{tt}(w) \geqq k$.

4.3 LeMMA B. If $c \geqq 1$, then $\left[M_{(c)}, p G\right] \geqq M_{(c+1)}$.

4.4 LeMma C. If $c \geqq 2$ and $e \geqq 0$, then $M_{(c)} \geqq\left[M_{(c-1)}, e G\right]$.

4.5 LEMMA D. Let $\tau: G \rightarrow G$ be the endomorphism induced by the mapping $g_{j} \mapsto g_{j+2}$ for all $j$, and, for each $k \geqq 1$, let $\kappa_{k}: G \rightarrow G$ be the endomorphism induced by the mapping $g_{k} \mapsto g_{k}\left[g_{2}, g_{1}\right]$ and $g_{j} \mapsto g_{j}$ for all $j \neq k$. Let $w \in G^{\prime}$ and for each $i \in\{1,2, \cdots\}$ let $w^{(i)}=\left(w \tau \kappa_{i+2}\right)(w \tau)^{-1}$.

a) $w^{(i)}$ is special for all $i$.

b) If $w$ is non-trivial, then so is $w^{(i)}$ for at least one value of $i$.

c) If $w$ is non-trivial, $c=\min \left(\operatorname{comp}\left(w^{(i)}\right) \mid i=1,2, \cdots\right)$ and

$$
d=\max (0, w t(w)-c p) \text {, }
$$

then $w \in\left[M_{(c)}, d G\right]$.

4.6 Lemma E. If $w$ is a non-trivial special element of $G^{\prime}$ with $\operatorname{comp}(w)=c$ and if $W$ is the fully invariant closure of $\{w\}$, then $W \geqq\left[M_{(c)}, e G\right]$ for some $e \geqq 0$.

4.7 LEMma F. $M_{(p)} \geqq G^{p^{2}} \cap G^{\prime}$. 
The remainder of this section is devoted to the proof of Theorem 1.1 on the basis of Lemmas A-F.

4.8 Notation. Let lat $(G)$ be the lattice of fully invariant subgroups of $G$ with respect to the inclusion ordering. If $U \in \operatorname{lat}(G)$, then let $\operatorname{id}(U)$ denote the ideal in lat $(G)$ generated by $U$; i.e. $\operatorname{id}(U)=\{V \in \operatorname{lat}(G) \mid V \leqq U\}$. In addition, let $\operatorname{id}^{*}(U)=\operatorname{id}(U) \backslash\{\{1\}\}$. Lastly, for any element $w \in G$, let $\langle w\rangle$ denote the fully invariant closure of $w$ in $G$.

4.9 Lemma. If $w \in G^{\prime}, w \neq 1$, then there exists an $e \geqq 1$ such that $M_{(c)} \geqq\langle w\rangle \geqq\left[M_{(c)}, e G\right]$, where $c=\min \left(\operatorname{comp}\left(w^{(i)}\right) \mid i=1,2, \cdots\right)$.

Proof. It is immediate from the definition that $w^{(i)} \in\langle w\rangle$ for all $i$. In particular, choosing an integer $i_{w}$ such that

$$
\operatorname{comp}\left(w^{\left(i_{w}\right)}\right)=\min \left(\operatorname{comp}\left(w^{(i)}\right) \mid i=1,2, \cdots\right)=c,
$$

it follows that $\langle w\rangle \geqq\left\langle w^{\left(i_{w}\right)}\right\rangle$ and hence, from Lemma E, that $\langle w\rangle \geqq\left[M_{(c)}, e G\right]$ for some large enough $e$. On the other hand, Lemma $\mathrm{D}$ specifies an integer $d$ such that $w \in\left[M_{(c)}, d G\right]$, and so, a fortiori, $w \in M_{(c)}$. Hence $M_{(c)} \geqq\langle w\rangle$ and the lemma is proved.

4.10 Lemma. Let $W \in \mathrm{id}^{\#}\left(G^{\prime}\right)$. Then there exist positive integers $c$,e such that $M_{(c)} \geqq W \geqq\left[M_{(c)}, e G\right]$.

PRoof. Let $\left\{w_{\lambda} \mid \lambda \in \Lambda\right\}$ be the complete set of nontrivial elements of $W$. By 4.9 , to each $\lambda \in \Lambda$ there exist $c_{\lambda}, e_{\lambda}$ such that $M_{\left(c_{\lambda}\right)} \geqq\left\langle w_{\lambda}\right\rangle \geqq\left[M_{\left(c_{\lambda}\right)}, e_{\lambda} G\right]$, and since $W=\cup_{\lambda \in \Lambda}\left\langle w_{\lambda}\right\rangle$ it follows that $\cup_{\lambda \in \Lambda} M_{\left(c_{\lambda}\right)} \geqq W \geqq \cup_{\lambda \in \Lambda}\left[M_{\left(c_{\lambda}\right)}, e_{\lambda} G\right]$. Now choose $\bar{\lambda} \in \Lambda$ such that $c_{\bar{\lambda}}=\min \left(c_{\lambda} \mid \lambda \in \Lambda\right)$ and write $c=c_{\bar{\lambda}}$ and $e=e_{\bar{\lambda}}$. Then, clearly, $M_{(c)} \geqq W \geqq\left[M_{(c)}, e G\right]$.

4.11 Lemma. If $w \in M_{(k)} \cap G^{\prime}, k \geqq 1$, then $\min \left(\operatorname{comp}\left(w^{(i)}\right) \mid i=1,2, \cdots\right) \geqq k$.

Proof. If $w=1$ the lemma is immediate, so assume $w \neq 1$. Then by 4.9 there exists an $e$ such that $\langle w\rangle \geqq\left[M_{\left(k^{\prime}\right)}, e G\right]$, where $k^{\prime}=\min \left(\operatorname{comp}\left(w^{(i)}\right) \mid i=1,2, \cdots\right)$. From this it follows that $M_{(k)} \geqq\left[M_{\left(k^{\prime}\right)}, e G\right]$, but unless $k^{\prime} \geqq k$ this contradicts Lemma $\mathrm{C}$.

4.12 Lemma. $\left[M_{(c)}, e G\right] \geqq M_{(c)} \cap G_{(c p+e)}$ for all positive integers $c$ and $e$.

Proof. It is sufficient to show that every non-trivial element of $M_{(c)} \cap G_{(c p+e)}$ is a member of $\left[M_{(\mathfrak{c})}, e G\right]$. So let $w$ be any such element. Then by Lemma $\mathrm{A}$ and 4.11 there exist non-negative $a_{1}$ and $a_{2}$ such that $w t(w)=c p+e+a_{1}$ and $\min \left(\operatorname{comp}\left(w^{(i)}\right) \mid i=1,2, \cdots\right)=c+a_{2}$. Hence by Lemma $\mathrm{D} w \in\left[M_{\left(c+a_{2}\right)}, d G\right]$ where $d=\max \left(0, c p+e+a_{1}-\left(c+a_{2}\right) p\right)=\max \left(0, e+a_{1}-a_{2} p\right)$. Now it follows from Lemma B that $\left[M_{\left(c+a_{2}\right)}, d G\right] \leqq\left[M_{(c)},\left(d+a_{2} p\right) G\right]$ and thus $w \in\left[M_{(c)}, d^{\prime} G\right]$ where 


$$
\begin{aligned}
d^{\prime}=d+a_{2} p & =\max \left(0, e+a_{1}-a_{2} p\right)+a_{2} p \\
& =\max \left(a_{2} p, e+a_{1}\right) \\
& \geqq e+a_{1} \geqq e
\end{aligned}
$$

This shows that $w \in\left[M_{(c)}, e G\right]$, as required.

4.13 LEMma. If $W \in \mathrm{id}^{\#}\left(G^{\prime}\right)$ then there exist integers $c \geqq 1$ and $d \geqq 2$ such that $W=M_{(c)} \cap L$ where $L \in$ lat $(G)$ with $L \geqq G_{(d)}$. Moreover if $W=M_{\left(c^{*}\right)} \cap L^{*}$ is any other such expression for $W\left(\right.$ say with $\left.L^{*} \geqq G_{\left(d^{*}\right)}\right)$ then $c=c^{*}$.

ProOF. By 4.10 and 4.12 there exist positive integers $c$ and $e$ such that $M_{(c)} \geqq W \geqq M_{(c)} \cap G_{(c p+e)}$. Setting $d=c p+e$ and $L=W . G_{(d)}$ this gives

$$
W=W\left(M_{(c)} \cap G_{(d)}\right)=M_{(c)} \cap W . G_{(d)}=M_{(c)} \cap L,
$$

the middle equality holding because of the modularity of $\operatorname{lat}(G)$. Now suppose

$$
M_{(c)} \cap L=M_{(c *)} \cap L^{*},
$$

and assume without loss of generality that $c^{*} \geqq c$. If in fact $c^{*}>c$, then, clearly, it follows that

$$
M_{\left(c^{*}\right)} \geqq M_{(c)} \cap G_{(d)} \geqq\left[M_{(c)}, d G\right] \geqq\left[M_{\left(c^{*}-1\right)}, d G\right],
$$

and this contradicts Lemma $\mathrm{C}$. Thus $c=c^{*}$.

4.14 LEMMA. $M_{(c)}=M_{(c)} \cdot G^{p^{2}} \cap G^{\prime}$ for all $c \in\{2, \cdots, p\}$.

Proof. Let $c \in\{2, \cdots, p\}$. Then $G^{\prime} \geqq M_{(c)} \geqq M_{(p)}$, and $M_{(p)} \geqq G^{p^{2}} \cap G^{\prime}$ by Lemma $F$. Hence, using modularity,

$$
M_{(c)}=M_{(c)} \cdot\left(G^{p^{2}} \cap G^{\prime}\right)=M_{(c)} \cdot G^{p^{2}} \cap G^{\prime} .
$$

4.15 LEMMA. $G^{p^{2}}=M_{(p)} \cdot G^{p^{2}} \cap G_{(d)} \cdot G^{p^{2}}$ for some $d$.

Proof. It is easy to check by routine induction on $r$ that if $a \in G, b \in G^{\prime}$ and $r \geqq 1$, then

$$
(a b)^{r}=a^{r} \prod_{i=1}^{r}[b,(i-1) a]^{\left(\begin{array}{c}
r \\
i
\end{array}\right)} .
$$

In particular, setting $a=g_{3}, b=\left[g_{2}, g_{1}\right]$ and $r=p^{2}$, and noting that $G^{\prime}$ has exponent $p$ and that $\left({ }^{p^{2}}\right) \equiv 0 \bmod p$ for all $i \in\left\{1, \cdots, p^{2}-1\right\}$, this shows that

$$
\left(g_{3}\left[g_{2}, g_{1}\right]\right)^{p^{2}}=g_{3}^{p^{2}}\left[g_{2}, g_{1},\left(p^{2}-1\right) g_{3}\right],
$$

and it follows that $w=\left[g_{2}, g_{1},\left(p^{2}-1\right) g_{3}\right] \in G^{p^{2}}$. By inspection, $w$ is special and has $p$-complexity $p$, so by Lemma $\mathrm{E}\langle w\rangle \geqq\left[M_{(p)}, e G\right]$ for some $e \geqq 0$. 
Setting $d=p^{2}+e$ it follows from 4.12 that $G^{p^{2}} \geqq M_{(p)} \cap G_{(d)}$. But by 4.14 $M_{(p)}=M_{(p)} \cdot G^{p^{2}} \cap G^{\prime}$, and so, using modularity,

$$
\begin{aligned}
G^{p^{2}}=G^{p^{2}} \cdot\left(M_{(p)} \cap G_{(d)}\right) & =G^{p^{2}}\left(M_{(p)} \cdot G^{p^{2}} \cap G^{\prime} \cap G_{(d)}\right) \\
& =M_{(p)} \cdot G^{p^{2}} \cap G_{(d)} \cdot G^{p^{2}} .
\end{aligned}
$$

4.16 Lemma. a) $\mathfrak{I}_{1}=\mathfrak{A}_{p} \mathfrak{U}_{p}$. In particular, $\mathfrak{I}_{1}$ is non-nilpotent.

b) If $\mathfrak{W}$ is a non-nilpotent proper subvariety of $\mathfrak{U}_{p} \mathfrak{A}_{p^{2}}$, and $\mathfrak{W}$ has exponent $p^{3}$, then there exists a unique $k \in\{1,2, \cdots\}$ such that $\mathfrak{B}=\mathfrak{I}_{k} \vee \mathfrak{L}$ for some nilpotent variety $\mathbb{Q}$.

c) $\mathfrak{I}_{p} \mathfrak{A}_{p^{2}} \wedge \mathfrak{B}_{p^{2}}=\mathfrak{I}_{p-1} \vee \mathfrak{L}_{0}$ for some nilpotent variety $\mathfrak{L}_{0}$.

Proof. a) By definition, $\mathfrak{J}_{1}=\mathfrak{A}_{p} \mathfrak{A}_{p^{2}} \wedge \mathfrak{U}_{p} \wedge \mathfrak{B}_{p^{2}}$, so it is clear that $\mathfrak{I}_{1} \geqq \mathfrak{A}_{p} \mathfrak{A}_{p}$. On the other hand, it is easy to check that the five words $[[x, y],[z, w]],[x, y]^{p},\left[x^{p}, y^{p}\right],\left[x, y, z^{p}\right]$ and $x^{p^{2}}$ form a basis for the laws of $\mathfrak{A}_{p} \mathfrak{A}_{p}$, and since the first two of these words are laws of $\mathfrak{U}_{p} \mathfrak{A}_{p^{2}}$, the third and fourth are laws of $\mathfrak{U H}_{p}$ and the fifth is a law of $\mathfrak{B}_{p^{2}}$, it follows that $\mathfrak{A}_{p} \mathfrak{A}_{p} \geqq \mathfrak{I}_{1}$. Thus $I_{1}=\mathfrak{A}_{p} \mathfrak{A}_{p}$. It is well-known that $\mathfrak{A}_{p} \mathfrak{A}_{p}$ is non-nilpotent (see 24.34 in [11], for example). That $\mathfrak{J}_{1}$ is non-nilpotent can also be proved directly from 4.13 and 4.14 .

b) For $k=1,2, \cdots$ let $I_{k}$ be the $\mathfrak{I}_{k}$-subgroup of $G$ :

$$
I_{k}=I_{k}(G)= \begin{cases}M_{(k+1)} \cdot G^{p^{2}} & \text { for } k \in\{1, \cdots, p-1\} \\ M_{(k+1)} & \text { for } k \geqq p .\end{cases}
$$

Let $\mathfrak{M}$ be as described in the statement of the lemma and let $W$ be the $\mathfrak{B}$-subgroup of $G: W=W(G)$. The fact that $\mathfrak{B}$ has exponent $p^{3}$ implies that $\mathfrak{B} \geqq \mathfrak{A}_{p^{3}}$ and therefore that $W \leqq A_{p^{3}}(G)=A(G)=G^{\prime}$. Since $\mathfrak{B}$ is a proper subvariety of $\mathfrak{A}_{p} \mathfrak{A}_{p^{2}}$, this means that $W \in \mathrm{id}^{\#}\left(G^{\prime}\right)$. Hence by 4.13 there exist integers $c \geqq 1$ and $d \geqq 2$ such that $W=M_{(c)} \cap L_{1}$ where $L_{1} \in \operatorname{lat}(G)$ with $L_{1} \geqq G_{(d)}$. Since $M_{(1)} \geqq G^{\prime} \geqq G_{(d)}$, it is clear that $c \neq 1$, for otherwise $W \geqq G_{(d)}$ contrary to the assumption that $\mathfrak{B}$ is non-nilpotent. Also if $c \in\{2, \cdots, p\}$, then by 4.14 $M_{(c)}=M_{(c)} . G^{p^{2}} \cap G^{\prime}$, and so in this case $W$ can also be expressed in the form $W=M_{(c)} . G^{p^{2}} \cap L_{2}$, where $L_{2}=L_{1} \cap G^{\prime} \geqq G_{(d)}$. It is thus established that $W=I_{k} \cap L$ for some $k \in\{1,2, \cdots\}$ and some $L \in \operatorname{lat}(G)$ with $L \geqq G_{(d)}$ for some $d$. But this means that $\mathfrak{B}=\mathfrak{I}_{k} \vee \mathfrak{L}$, where $\mathfrak{Q}$ is the variety corresponding to $L$ and is therefore nilpotent. If also $\mathfrak{W}=\mathfrak{I}_{k^{*}} \vee \mathfrak{L}^{*}$ with $\mathfrak{L}^{*}$ nilpotent, then, since $I_{k} \cap G^{\prime}=M_{(k+1)}$ for all $k=1,2, \cdots$,

$$
M_{(k+1)} \cap L=I_{k} \cap L \cap G^{\prime}=I_{k^{*}} \cap L^{*} \cap G^{\prime}=M_{\left(k^{*+1}\right)} \cap L^{*},
$$

and by the second part of 4.13 this implies that $k=k^{*}$. Thus $k$ is uniquely determined by $\mathfrak{W}$.

c) This is an immediate consequence of 4.15 . 
Theorem 1.1 is an almost immediate consequence of this last lemma. Since by Meier-Wunderli's result in [10] every metabelian variety of exponent $p$ is nilpotent, it is clear that all that remains to prove is that statement b) of 4.16 remains valid when "exponent $p^{3}$ ", is replaced by "exponent $p^{2}$ ". So let $\mathfrak{W}$ be a non-nilpotent exponent $p^{2}$ subvariety of $\mathfrak{A}_{p} \mathfrak{I}_{p}{ }^{2}$. Then, since

$$
\mathfrak{W}=\mathfrak{W} \vee \mathfrak{A}_{p^{2}}=\mathfrak{W} \vee\left(\mathfrak{A}_{p^{3}} \wedge \mathfrak{B}_{p^{2}}\right)=\left(\mathfrak{W} \vee \mathfrak{U}_{p^{3}}\right) \wedge \mathfrak{B}_{p^{2}},
$$

parts b) and c) of 4.16 ensure the existence of a $k^{\prime} \in\{1,2, \cdots\}$ and nilpotent varieties $\mathfrak{P}^{\prime}$ and $\mathfrak{L}_{0}$ such that

$$
\mathfrak{W}=\left(\mathfrak{I}_{k^{\prime}} \vee \mathfrak{L}^{\prime}\right) \wedge\left(\mathfrak{I}_{p-1} \vee \mathfrak{L}_{0}\right)=\mathfrak{I}_{k} \vee \mathfrak{L},
$$

where $k=\min \left(k^{\prime}, p-1\right)$ and $\mathfrak{L}$ is either $\mathfrak{L}_{0} \wedge\left(\mathfrak{I}_{k} \vee \mathfrak{L}^{\prime}\right)$ or $\mathfrak{L}^{\prime} \wedge\left(\mathfrak{I}_{p-1} \vee \mathfrak{L}_{0}\right)$ but in any case is nilpotent. If also $\mathfrak{W}=\mathfrak{I}_{k^{*}} \vee \mathfrak{L}^{*}$, then

$$
\mathfrak{W} \vee \mathfrak{A}_{p^{3}}=\mathfrak{I}_{k} \vee\left(\mathfrak{L} \vee \mathfrak{A}_{p^{3}}\right)=\mathfrak{I}_{k} \vee\left(\mathfrak{I}^{*} \vee \mathfrak{A}_{p^{3}}\right)
$$

and so $k=k^{*}$.

\section{Proof of Lemma $A$}

The fact that $G^{\prime}$ has exponent $p$, coupled with the fact that $\left(\begin{array}{c}p_{i}^{2} \\ i\end{array}\right) \equiv 0 \bmod p$ for all $i \in\left\{1, \cdots, p^{2}-1\right\}$, leads to the following lemma, which helps to simplify commutator calculus in $G$ :

5.1 LEMMA. Let $(a, b, \delta)$ be a pseudo-commutator in $G$.

a) Any one of the following conditions implies that $[a, b, \delta]=1$.

(i) $\delta(a) \geqq p^{2}+1$.

(ii) $\delta(b) \geqq p^{2}+1$.

(iii) $\delta(c) \geqq p^{2}$ for some $c \notin\{a, b\}$.

(iv) $\delta(a)=\delta(b)=p^{2}$.

b) If $\delta(b)=p^{2}$ then $[a, b, \delta]=[c, b, \delta]$ for all $c \in \operatorname{supp} \delta \backslash\{b\}$.

Proof. a) If $u \in G^{\prime}$ and $v \in G$ then

$$
1=\left[u, v^{p^{2}}\right]=\prod_{i=1}^{p^{2}}[u, i v]^{\left(\begin{array}{c}
p^{2} \\
i
\end{array}\right)}=\left[u, p^{2} v\right],
$$

so any one of the conditions (i) -(iii) implies $[a, b, \delta]=1$. That condition (iv) also implies $[a, b, \delta]=1$ is immediate from part c) of Lemma 2.3.

b) This is immediate from $2.3 \mathrm{~d}$ ).

Lemma 5.1 is needed in the proof of

5.2 LEMMA. If $\left(a_{1}, a_{2}, \delta\right)$ is a (not necessarily basic) pseudo-commutator in $G$ with $\operatorname{supp} \delta \subseteq \mathfrak{g}$ and non-trivial value, then $\operatorname{wt}\left(\left[a_{1}, a_{2}, \delta\right]\right)=\operatorname{wt}\left(\left(a_{1}, a_{2}, \delta\right)\right)$. 
Proof. Let $\operatorname{supp} \delta=\left\{a_{1}, \cdots, a_{s}\right\}$, where $s \geqq 2$ since $a_{1} \neq a_{2}$. By $\left.5.1 \mathrm{a}\right)$ $\delta\left(a_{1}\right) \leqq p^{2} ; \delta\left(a_{2}\right) \leqq p^{2} ; \delta\left(a_{j}\right)<p^{2}$ for $j \in\{3, \cdots, s\}$; and $\delta\left(a_{1}\right)$ and $\delta\left(a_{2}\right)$ are not both $p^{2}$.

There are two cases to consider.

(i) Suppose $\min \left\{a_{1}, \cdots, a_{s}\right\}=a_{i}$, where $a_{1} \neq a_{i} \neq a_{2}$. By the Jacobi identity $\left[a_{1}, a_{2}, \delta\right]=\left[a_{1}, a_{i}, \delta\right]\left[a_{2}, a_{i}, \delta\right]^{-1}$, and it follows from the restrictions on the values of the $\delta\left(a_{j}\right)$ that the pseudo-commutator $\left(a_{1}, a_{i}, \delta\right)$ is basic unless $\delta\left(a_{2}\right)=p^{2}$, in which case $\left[a_{1}, a_{i}, \delta\right]=1$ (by $5.1 \mathrm{a}$ ), case (iii)). A similar statement holds for $\left(a_{2}, a_{i}, \delta\right)$, so the expression in normal form for $\left[a_{1}, a_{2}, \delta\right]$ involves only the values of basic pseudo-commutators with degree function $\delta$. Thus $\operatorname{wt}\left(\left[a_{1}, a_{2}, \delta\right]\right)$ $=\sum_{j=1}^{s} \delta\left(a_{j}\right)=\operatorname{wt}\left(\left(a_{1}, a_{2}, \delta\right)\right)$.

(ii)The alternative case occurs when $\min \left\{a_{1}, \cdots, a_{s}\right\}$ is $a_{1}$ or $a_{2}$. In fact it may be assumed to be $a_{2}$, for clearly wt $\left(\left(a_{1}, a_{2}, \delta\right)\right)=\mathrm{wt}\left(\left(a_{2}, a_{1}, \delta\right)\right)$ and $\mathrm{wt}\left(\left[a_{1}, a_{2} \delta\right]\right)$ $=\operatorname{wt}\left(\left[a_{2}, a_{1}, \delta\right]^{-1}\right)=\operatorname{wt}\left(\left[a_{2}, a_{1}, \delta\right]\right)$. Similarly, if $\delta\left(a_{2}\right)=p^{2}$ then by $\left.5.1 \mathrm{~b}\right)$ it may be assumed that $\max \left\{a_{1}, \cdots, a_{s}\right\}=a_{1}$. But these assumptions imply that $\left(a_{1}, a_{2}, \delta\right)$ is basic, which means that there is nothing left to prove.

There is an immediate corollary:

5.3 Corollary. If $\left(a_{1}, a_{2}, \delta\right)$ is a pseudo-commutator in $G$ with $\operatorname{supp} \delta \subseteq \mathfrak{g}$ and non-trivial value, then

for all $a \in \mathfrak{g}$.

$$
\mathrm{wt}\left(\left[\left[a_{1}, a_{2}, \delta\right], a\right]\right) \geqq \operatorname{wt}\left(\left[a_{1}, a_{2}, \delta\right]\right)+1
$$

More generally...

5.4 Lemma. If $w \in G^{\prime}, w \neq 1$, and $v \in G$, then $\operatorname{wt}([w, v]) \geqq \operatorname{wt}(w)+1$.

Proof. For $v=1$ the result is trivial, so assume $v \neq 1$. Then, since $G$ has finite exponent, $v=a_{1} a_{2} \cdots a_{s}$ for some $a_{1}, \cdots, a_{s} \in \mathfrak{g}$ (not necessarily all distinct). The proof uses induction on $s$.

To deal with the case $s=1$ first express $w$ in normal form by $w=b_{1}^{e_{1}} \cdots b_{t}^{e_{t}}$ say, and note that $\omega>\operatorname{wt}\left(b_{j}\right) \geqq \operatorname{wt}(w)$ for each $j \in\{1, \cdots, t\}$. Then

$$
\begin{aligned}
& \operatorname{wt}\left(\left[w, a_{1}\right]\right)=\operatorname{wt}\left(\left[b_{1}, a_{1}\right]^{e_{1}} \cdots\left[b_{t}, a_{1}\right]^{e_{t}}\right) \\
& \geqq \min _{j}^{j}\left(\operatorname{wt}\left(\left[b_{j}, a_{1}\right]\right) \mid j=1, \cdots, t\right) \\
& \geqq \min _{j}\left(\operatorname{wt}\left(b_{j}\right)+1 \mid j=1, \cdots, t\right)(\text { by } 5.3) \\
&=\min _{j}\left(\operatorname{wt}\left(b_{j}\right) \mid j=1, \cdots, t\right)+1=\operatorname{wt}(w)+1 .
\end{aligned}
$$

The inductive step is routine, and is therefore omitted.

Since $G_{(k+1)}=\left[G_{(k)}, G\right]$ for all $k \geqq 1$, Lemma A (4.2) easily follows from 5.4 by induction on $k$. 


\section{Proof of Lemma B}

6.1 Notation. For $c \geqq 1$ and $e \geqq 0$ let $U(c, e)$ and $V(c, e)$ be the verbal subgroups of $G$ defined by

$$
U(c, e)=\left\{\left[y_{1}^{p}, \cdots, y_{c}^{p}, z_{1}, \cdots, z_{e}\right]\right\}(G)
$$

and

$$
V(c, e)=\left\{\left[x_{1}, x_{2}, y_{2}^{p}, \cdots, y_{c}^{p}, z_{1}, \cdots, z_{e}\right]\right\}(G) .
$$

The following lemma facilitates the proof of Lemma B (4.3) and will also be used in $\S 9$ to help with the proof of Lemma $E$ (4.6):

6.2 Lemma. For all $c \geqq 1$ and all $e \geqq 0,\left[M_{(c)}, e G\right]=U(c, e) . V(c, e)$.

Proof. Clearly

$\left[M_{(c)}, e G\right]=\operatorname{gp}\left(\left[m_{1}, \cdots, m_{c}, w_{1}, \cdots, w_{e}\right] \mid m_{1}, \cdots, m_{c} \in M ; w_{1}, \cdots, w_{e} \in G\right)$,

so the inclusion $\left[M_{(c)}, e G\right] \geqq U(c, e) . V(c, e)$ is immediate. Since any $m \in M$ can be expressed in the form $m=v^{p} v^{\prime}$ for some $v \in G$ and $v^{\prime} \in G^{\prime}$, it is sufficient for the reverse inclusion to prove that

$$
\left[v_{1}^{p} v_{1}^{\prime}, \cdots, v_{c}^{p} v_{c}^{\prime}, w_{1}, \cdots, w_{e}\right] \in U(c, e) . V(c, e)
$$

for all $v_{1}, \cdots, v_{c}, w_{1}, w_{e} \in G$ and all $v_{1}{ }^{\prime}, \cdots, v_{c}{ }^{\prime} \in G^{\prime}$. The proof of this fact is a routine exercise in commutator calculus.

To prove Lemma B (4.3) first note that for any $u, v \in G$

$$
\left[u, v^{p}\right]=\prod_{i=1}^{p}[u, i v]^{\left(\begin{array}{l}
p \\
i
\end{array}\right)}=[u, p v] .
$$

Hence

$$
\begin{aligned}
U(c, p) & =\operatorname{gp}\left(\left[v_{1}^{p}, \cdots, v_{c}^{p}, w_{1}, \cdots, w_{p}\right] \mid v_{1}, \cdots, v_{c}, w_{1}, \cdots, w_{p} \in G\right) \\
& \geqq \operatorname{gp}\left(\left[v_{1}^{p}, \cdots, v_{c}^{p}, p v_{c+1}\right] \mid v_{1}, \cdots, v_{c+1} \in G\right) \\
& =\operatorname{gp}\left(\left[v_{1}^{p}, \cdots, v_{c+1}^{p}\right] \mid v_{1}, \cdots, v_{c+1} \in G\right)=U(c+1,0),
\end{aligned}
$$

and similarly $V(c, p) \geqq V(c+1,0)$. So, using 6.2 ,

$$
\left[M_{(c)}, p G\right]=U(c, p) . V(c, p) \geqq U(c+1,0) \cdot V(c+1,0)=M_{(c+1)},
$$

which proves Lemma B (4.3).

\section{Proof of Lemma $C$}

The ideas for this section are due to L. G. Kovács.

Let $c \geqq 2$ and $e \geqq 1$ be chosen arbitrarily and then fixed. Let $G^{*}$ be the wreath product of finite $p$-groups defined by $G^{*}=R \operatorname{wr}(S \times T)$, where 


$$
\begin{aligned}
& R=\operatorname{gp}\left(r \mid r^{p}=1\right) \\
& S=S_{1} \times \cdots \times S_{c-2} ; S_{i}=\operatorname{gp}\left(s_{i} \mid s_{i}^{p^{2}}=1\right), i \in\{1, \cdots, c-2\} \\
& T=T_{0} \times \cdots \times T_{e} ; T=\operatorname{gp}\left(t_{j} \mid t_{j}^{p}=1\right), j \in\{0, \cdots, e\},
\end{aligned}
$$

and of course $S=\{1\}$ if $c=2$. The base group of $G^{*}$ will be denoted by $K$, and is to be considered as consisting of all functions from $S \times T$ into $R$, with multiplication defined component-wise. Additionally, for each $i \in\{1, \cdots, c-2\}$, $j \in\{0, \cdots, e\}$, notation will be abused to the extent of considering $S_{i}$ and $T_{j}$ (and so also $S$ and $T$ ) as subgroups of $G^{*}$ via the standard embedding.

Denote the $\mathfrak{A}_{p}$-subgroup of $G^{*}$ by $M^{*} ; M^{*}=A_{p}\left(G^{*}\right)$. Two facts about $M^{*}$ will be needed, and both follow from results of Liebeck [8].

\subsection{Lemma. $M^{*}{ }_{((c-2)(p-1)+2)}=\{1\}$.}

Proof. Clearly $M^{*} \leqq K . S^{p}=\bar{M}^{*}$ say. Now from the proof of 22.14 in [11] it follows that $\bar{M}^{*} \cong R^{\dagger}$ wr $S^{p}$ where $R^{\dagger}$ denotes the direct product of $\left|(S \times T) / S^{p}\right|$ copies of $R$. Thus, from [8] Theorem 5.1, $\bar{M}^{*}$ has nilpotency class $(c-2)(p-1)+1$ and the conclusion follows.

7.2 Lemma. If $k \in K$ is defined by $k(1)=r$ and $k(v)=1$ for all $v \in(S \times T) \backslash\{1\}$, then

$$
\left[k, t_{0},(p-1) s_{1}^{p}, \cdots,(p-1) s_{c-2}^{p}, t_{1}, \cdots, t_{e}\right] \neq 1 .
$$

Proof. It follows from part (a) of the proof of Theorem 5.1 in [8] that

$$
\left[k,\left(p^{2}-1\right) s_{1}, \cdots,\left(p^{2}-1\right) s_{c-2},(p-1) t_{0}, \cdots,(p-1) t_{e}\right] \neq 1
$$

and hence, a fortiori, that

$$
\left[k,(p-1) p s_{1}, \cdots,(p-1) p s_{c-2}, t_{0}, \cdots, t_{e}\right] \neq 1 .
$$

Since $[u, p v]=\left[u, v^{p}\right]$ for all $u, v \in G^{*}$, this is equivalent to

$$
\left[k,(p-1) s_{1}^{p}, \cdots,(p-1) s_{c-2}^{p}, t_{0}, t_{1}, \cdots, t_{e}\right] \neq 1 .
$$

This establishes the lemma, for by 5.7 in [8] an alteration to the order of entries occurring after $k$ leaves the commutator unchanged.

Since $G^{*} \in \mathfrak{A}_{p} \mathfrak{A}_{p^{2}}$ it is clear that Lemma $C$ (4.4) is an immediate consequence of

7.3 Lemma. $M_{(c)}^{*}\left[M_{(c-1)}^{*}, e G^{*}\right]$.

Proof. With $k$ defined as above let $w=\left[k, t_{0}, s_{1}^{p}, \cdots, s_{c-2}^{p}, t_{1}, \cdots, t_{e}\right]$. Since clearly $w \in\left[M^{*}{ }_{(c-1)}, e G^{*}\right]$, the lemma will be proved once it is shown that $w \notin M^{*}{ }_{(c)}$. Suppose to the contrary that $w \in M^{*}{ }_{(c)}$. Then it follows that 


$$
\left[w,(p-2) s_{1}^{p}, \cdots,(p-2) s_{c-2}^{p}\right] \in M^{*}{ }_{(c+(c-2)(p-2))},
$$

i.e. that

$$
\left[k, t_{0},(p-1) s_{1}^{p}, \cdots,(p-1) s_{c-2}^{p}, t_{1}, \cdots, t_{e}\right] \in M^{*}{ }_{((c-2)(p-1)+2)} .
$$

But from 7.1 and 7.2 this is impossible.

\section{Proof of Lemma D}

It is clear that for each (fixed) $i$ the mapping $w \mapsto w^{(i)}$ of $G^{\prime}$ into itself is an endomorphism of $G^{\prime}$. The first objective, therefore, will be to describe the effect of these endomorphisms of $G^{\prime}$ on members of the basis $[\widetilde{B}]$ of $G^{\prime}$.

8.1 Notation. For each integer $i>0$ and each degree function $\delta$ on $G$ with $g_{i} \in \operatorname{supp} \delta$ let $\delta^{(i)}$ be the degree function on $G$ defined by

$$
\begin{aligned}
& \delta^{(i)}\left(g_{1}\right)=\delta^{(i)}\left(g_{2}\right)=1, \\
& \delta^{(i)}\left(g_{i+2}\right)=\delta\left(g_{i}\right)-1, \\
& \delta^{(i)}\left(g_{j}\right)=\delta\left(g_{j-2}\right) \text { for all } j \geqq 3, j \neq i+2, \text { and } \\
& \delta^{(i)}(a)=0 \text { for all } a \in G \backslash g .
\end{aligned}
$$

Note that $\left(g_{2}, g_{1}, \delta^{(i)}\right)$ is always a special pseudo-commutator in $G$.

A straightforward commutator calculation verifies the following formula:

8.2 LEMMA. If $(a, b, \delta)$ is a non-trivial pseudo-commutator in $G$ with $\operatorname{supp} \delta \subseteq \mathfrak{g}$ then

$$
[a, b, \delta]^{(i)}= \begin{cases}{\left[g_{2}, g_{1}, \delta^{(i)}\right]} & \text { if } a=g_{i}, \\ {\left[g_{2}, g_{1}, \delta^{(i)}\right]^{-1}} & \text { if } b=g_{i}, \\ 1 & \text { otherwise. }\end{cases}
$$

Now consider part a) of Lemma D (4.5). For $w=1$ it is a triviality, so assume $w$ to be expressed in normal form by $w=b_{1}^{e_{1}} \cdots b_{t}^{e_{t}}$. Clearly, $w^{(i)}=\left(b_{1}^{(i)}\right)^{\boldsymbol{e}_{1}} \cdots\left(b_{t}^{(i)}\right)^{\boldsymbol{e}_{t}}$ for all $i$, and since a product of special elements is special it is therefore sufficient to show that if $(a, b, \delta) \in \widetilde{B}$ then $[a, b, \delta]^{(i)}$ is special for all $i$. If $g_{i} \notin\{a, b\}$ then $[a, b, \delta]^{(i)}=1$ by 8.2 , so suppose $a=g_{i}$. Then, again by $8.2,[a, b, \delta]^{(i)}=\left[g_{2}, g_{1}, \delta^{(i)}\right]$. Since $(a, b, \delta)$ is basic, it is clear from the definition of $\delta^{(i)}$ that $\left(g_{2}, g_{1}, \delta^{(i)}\right)$ is also basic unless $\delta(b)=p^{2}$. However, in the event of the latter contingency, case (iii) of 5.1 a) gives that then $\left[g_{2}, g_{1}, \delta^{(i)}\right]=1$, so that possibility can be dismissed. Thus $[a, b, \delta]^{(i)}=[\tilde{b}]$, where $\tilde{b}=\left(g_{2}, g_{1}, \delta^{(i)}\right)$ is basic and special; i.e. $[a, b, \delta]^{(i)}$ is special. The case $b=g_{i}$ is handled similarly, and part a) of Lemma D is proved.

It is well-known that in any product variety the free group of countably infinite rank has trivial centre. (By 22.22, 22.32 and 24.23 in [11], any product 
variety is generated by a group with trivial centre. Now use the fact that in a relatively free group of countably infinite rank the centre is verbal.) Thus $G$ has trivial centre and therefore part b) of Lemma $D(4.5)$ is an immediate consequence of the following result:

8.3 Lemma. Let $w \in G^{\prime}$ and let $v \in G$. If for each integer $i>0$ the image of $u \in G$ under the endomorphism of $G$ induced by the mapping

$$
g_{1} \mapsto v, g_{2} \mapsto g_{i} \text { and } g_{j} \mapsto g_{j-2} \text { for all } j \geqq 3
$$

is denoted by $u^{(v, i)}$, then there exists an $s_{0}=s_{0}(w)$ such that

$$
\prod_{i=1}^{s} w^{(i)(v, i)}=[w, v]
$$

for all $s\rangle s_{0}$. In particular, $[w, v] \in\left\langle w^{(i)} \mid i=1,2, \cdots\right\rangle$.

PROOF. It is clearly sufficient to prove the result for $w=[\tilde{b}]$ where $\tilde{b}=\left(g_{k}, g_{l}, \delta\right) \in \widetilde{B}$.

It is no trouble to check that if $\delta$ is any degree function on $G$ with $g_{i} \in \operatorname{supp} \delta$ then

$$
\left[g_{2}, g_{1}, \delta^{(i)}\right]^{(v, i)}=\left[g_{i}, v, \delta+\chi_{v}\right],
$$

where $\chi_{v}$ is the characteristic function $\chi_{v}(v)=1, \chi_{v}(a)=0$ for all $a \neq v$. Setting $s_{0}=\max (k, l)=k$, and using 8.2 , it follows that for all $s \geqq s_{0}$

$$
\prod_{i=1}^{s} w^{(i)(v, i)}=\left[g_{l}, v, \delta+\chi_{v}\right]^{-1}\left[g_{k}, v, \delta+\chi_{v}\right] .
$$

Hence, by the Jacobi identity,

$$
\prod_{i=1}^{s} w^{(i)(v, i)}=\left[g_{k}, g_{l}, \delta+\chi_{v}\right]=\left[\left[g_{k}, g_{l}, \delta\right], v\right]=[w, v] .
$$

It remains to prove part c) of Lemma $D(4.5)$, and unfortunately this is a rather tedious business. Firstly:

8.4 Notation. For any $w \in G^{\prime}$ denote $\min _{i}\left(\operatorname{comp}\left(w^{(i)}\right) \mid i=1,2, \cdots\right)$ by $\operatorname{mic}(w)$.

Call a non-trivial element $w$ of $G^{\prime}$ well-behaved if $w \in\left[M_{(c)}, d G\right]$ where $c=\operatorname{mic}(w)$ and $d=\max (0, \mathrm{wt}(w)-c p)$. In this terminology part $c)$ of Lemma $\mathrm{D}$ (4.5) says that every non-trivial element of $G^{\prime}$ is well-behaved. The following lemma indicates how the task of proving this is reduced:

8.5 Lemma. If $w=\Pi_{j=1}^{s} w_{j} \neq 1$, where

(i) the $w_{j}$ are well-behaved members of $G^{\prime}$,

(ii) $\operatorname{wt}(w)=\min _{j}\left(\operatorname{wt}_{(}\left(w_{j}\right) \mid j=1, \cdots, s\right)$, and

(iii) $\operatorname{mic}(w)=\min _{j}\left(\operatorname{mic}\left(w_{j}\right) \mid j=1, \cdots, s\right)$,

then $w$ is well behaved. 
Proof. Set $c=\operatorname{mic}(w), d=\max (0, \mathrm{wt}(w)-c p), \quad c_{j}=\operatorname{mic}\left(w_{j}\right)$ and $d_{j}=\max \left(0, w \mathrm{t}\left(w_{j}\right)-c_{j} p\right)$. Condition (i) ensures that $w_{j} \in\left[M_{\left(c_{j}\right)}, d_{j} G\right]$, condition (ii) ensures that $\mathrm{wt}\left(w_{j}\right) \geqq \mathrm{wt}(w)$ and condition (iii) ensures that $c_{j} \geqq c$. Now by Lemma B (4.3)

$$
\left[M_{\left(c_{j}\right)}, d_{j} G\right]=\left[M_{\left(c+\left(c_{j}-c\right)\right)}, d_{j} G\right] \leqq\left[M_{(c)},\left(d_{j}+\left(c_{j}-c\right) p\right) G\right],
$$

so $w_{j} \in\left[M_{(c)}, d^{\prime} G\right]$, where

$$
\begin{aligned}
d^{\prime} & =d_{j}+\left(c_{j}-c\right) p=\max \left(0, \operatorname{wt}\left(w_{j}\right)-c_{j} p\right)+\left(c_{j}-c\right) p \\
& =\max \left(\left(c_{j}-c\right) p, \operatorname{wt}\left(w_{j}\right)-c p\right) \geqq \max (0, w \mathrm{t}(w)-c p)=d .
\end{aligned}
$$

Thus $w_{j} \in\left[M_{(c)}, d G\right]$ for each $j$, and consequently $w \in\left[M_{(c)}, d G\right]$. That is, $w$ is well-behaved.

It is to be noted that an arbitrary product $w=w_{1} \cdots w_{s} \neq 1$ with $w_{1}, \cdots, w_{s} \in G^{\prime}$ generally satisfies neither condition (ii) nor (iii) of the above lemma. Even if $w_{j}=b_{j}^{e_{j}}$ for $j=1, \cdots, s$ and $w$ is expressed in normal form by $w=b_{1}^{e_{1}} \cdots b_{s}^{e_{s}}$, then, although in that case condition (ii) is satisfied, condition (iii) may still not be satisfied; for example, if

$$
w=\left[g_{2}, p g_{1}, g_{3}\right]\left[g_{3}, p g_{1}, g_{2}\right]^{-1}
$$

then it is easy to check that $\operatorname{mic}(w)=2$ whereas

$$
\operatorname{mic}\left(\left[g_{2}, p g_{1}, g_{3}\right]\right)=\operatorname{mic}\left(\left[g_{3}, p g_{1}, g_{2}\right]^{-1}\right)=1
$$

Notice that in this example the relevant basic pseudo-commutators have the same degree function. (The pseudo-commutators concerned here are $\left(g_{2}, g_{1}, \delta\right)$ and $\left(g_{3}, g_{1}, \delta\right)$, where supp $\delta=\left\{g_{1}, g_{2}, g_{3}\right\}$ and $\delta\left(g_{1}\right)=p, \delta\left(g_{2}\right)=\delta\left(g_{3}\right)=1$.) It is convenient to give a name to elements of $G^{\prime}$ like the $w$ of this example. They will be called elementary: an elementary element of $G^{\prime}$ is one for which the basic pseudo-commutators involved in its expression in normal form have a common degree function. This common degree function will be called the degree of the elementary element. Clearly every element $w \in G^{\prime}$ is the product of its elementary parts; $w=\Pi_{i=1}^{s} w_{i}$ with each of $w_{1}, \cdots, w_{s}$ elementary and the degree functions of the $w_{i}$ distinct in pairs. The point of this is:

8.6 Lemma. Conditions (ii) and (iii) of 8.5 are always satisfied if $w_{1}, \cdots, w_{s}$ are the elementary parts of $w$.

Proof. Since condition (ii) is obviously satisfied under these circumstances it is only necessary to establish (iii).

For $j=1, \cdots, s$ let the degree function of $w_{j}$ be $\delta_{j}$. Using 8.2 and case (iii) of 5.1 a) it is easy to verify that for any $j \in\{1, \cdots, s\}$ and any $i \in\{1,2, \cdots\}$ the element $w_{j}^{(i)}$ is either trivial or is expressed in normal form by 


$$
w_{j}^{(i)}=\left[g_{2}, g_{1}, \delta_{j}^{(i)}\right]^{e_{j i}}
$$

for some $e_{j i} \neq=0 \bmod p$. Consequently, if $J_{i}$ denotes the set of those $j$ for which $w_{j}^{(i)} \neq 1$, the

$$
w^{(i)}=\prod_{j \in J_{i}}\left[g_{2}, g_{1}, \delta_{j}^{(i)}\right]^{e_{j i}}
$$

and as $\delta_{j}^{(i)} \neq \delta_{j^{\prime}}^{(i)}$ whenever $j \neq j^{\prime}$ this expresses $w^{(i)}$ in normal form. (Of course if $J_{i}$ is empty then $w^{(i)}=1$ ). Since $\operatorname{comp}(1)=\omega$ this shows that

$$
\operatorname{comp}\left(w^{(i)}\right)=\underset{j}{\min }\left(\operatorname{comp}\left(w_{j}^{(i)}\right) \mid j=1, \cdots, s\right),
$$

and from this condition (iii) of 8.5 follows.

In view of Lemmas 8.5 and 8.6, part c) of Lemma D (4.5) will follow when it is shown that every elementary element of $G^{\prime}$ is well-behaved. This latter result will presently be proved as Lemma 8.8 , but a preparatory result, Lemma 8.7 that follows now, is needed first.

8.7 LEMMA. Every element $w \in G^{\prime}$ whose expression in normal form is of the kind $w=b^{e}$ is well-behaved.

Proof. Choose $\tilde{b} \in \widetilde{B}$ and an integer $e \not 0 \bmod p$ arbitrarily, and set $w=[\tilde{b}]^{e}, c=\operatorname{mic}(w)$ and $d=\max (0, \mathrm{wt}(w)-c p)$. Since it is clear that $c$ and $d$ are independent of $e$, it may be assumed without loss of generality that $e=1$, for if $b \in\left[M_{(c)}, d G\right]$ then certainly $b^{e} \in\left[M_{(c)}, d G\right]$. Let $\tilde{b}=\left(a_{1}, a_{2}, \delta\right)$ with $\operatorname{supp} \delta=\left\{a_{1}, \cdots, a_{s}\right\}$ say (where, of course, each $\left.a_{j} \in \mathfrak{g}\right\}$, and for $j=1, \cdots, s$ write $a_{j}=g_{i_{j}}$ and $\delta\left(a_{j}\right)=q_{j} p+r_{j}$ with $0 \leqq r_{j}<p$. By employing Lemma 8.2 and the relevant definitions it may be verified that

$$
\begin{aligned}
c & =\underset{j}{\min }\left(\operatorname{comp}\left(\left[g_{2}, g_{1}, \delta^{\left(i_{j}\right)}\right] \mid j=1, \cdots, s\right)\right. \\
& = \begin{cases}\sum_{j=1}^{s} q_{j}+1 & \text { if } r_{1} \neq 0 \neq r_{2}, \\
\sum_{j=1}^{s} q_{j} & \text { otherwise. }\end{cases}
\end{aligned}
$$

Also, it follows straight from the definition of weight that

$$
\operatorname{wt}(w)=\operatorname{wt}(\tilde{b})=\sum_{j=1}^{s} \delta\left(a_{j}\right)=p \sum_{j=1}^{s} q_{j}+\sum_{j=1}^{s} r_{j} .
$$

It is now necessary to consider three cases, delimited according to the values of $r_{1}$ and $r_{2}$ :

Case 1: Assume that $r_{1} \neq 0 \neq r_{2}$. By making use of the fact that $[u, p v]=\left[u, v^{p}\right]$ for all $u, v \in G$, the element $w$ may be written in the form 


$$
w=\left[\left[\left[a_{1}, a_{2}\right], q_{1} a_{1}^{p}, \cdots, q_{s} a_{s}^{p}\right],\left(r_{1}-1\right) a_{1},\left(r_{2}-1\right) a_{2}, r_{3} a_{3}, \cdots, r_{s} a_{s}\right],
$$

and from this expression it is immediate that $w \in\left[M_{\left(c^{\prime}\right)}, d^{\prime} G\right]$, where $c^{\prime}=\sum_{i=1}^{s} q_{j}+1$ and $d^{\prime}=\sum_{j=1}^{s} r_{j}-2$. But by the remarks above $c^{\prime}=c$ and

$$
\begin{aligned}
d^{\prime} & =\operatorname{wt}(w)-p \sum_{j=1}^{s} q_{j}-2=\operatorname{wt}(w)-p(c-1)-2 \\
& =(w t(w)-c p)+(p-2) \geqq \max (0, \operatorname{wt}(w)-c p)=d,
\end{aligned}
$$

and consequently $\left[M_{\left(c^{\prime}\right)}, d^{\prime} G\right] \leqq\left[M_{(c)}, d G\right]$. Thus $w \in\left[M_{(c)}, d G\right]$; that is, $w$ is well-behaved.

Case 2. Assume that either $r_{1} \neq 0=r_{2}$ or $r_{1}=0 \neq r_{2}$. Then $w$ may be written either in the form

$w=\left[\left[\left[a_{1}, a_{2}\right], q_{1} a_{1}^{p},\left(q_{2}-1\right) a_{2}^{p}, q_{3} a_{3}^{p}, \cdots, q_{s} a_{s}^{p}\right],\left(r_{1}-1\right) a_{1},(p-1) a_{2}, r_{3} a_{3}, \cdots, r_{s} a_{s}\right]$

or in the form

$$
w=\left[\left[\left[a_{1}, a_{2}\right],\left(q_{1}-1\right) a_{1}^{p}, q_{2} a_{2}^{p}, \cdots, q_{s} a_{s}^{p}\right],(p-1) a_{1},\left(r_{2}-1\right) a_{2}, r_{3} a_{3}, \cdots, r_{s} a_{s}\right] .
$$

In any event, it is clear that $w \in\left[M_{\left(c^{\prime}\right)}, d^{\prime} G\right]$, where $c^{\prime}=\sum_{j=1}^{s} q_{j}$ and $d^{\prime}=\sum_{j=1}^{s} r_{j}+p-2$. Here again $c^{\prime}=c$, and

$$
\begin{gathered}
d^{\prime}=\mathrm{wt}(w)-p \sum_{j=1}^{s} q_{j}+p-2=(\mathrm{wt}(w)-c p)+(p-2) \\
\geqq \max (0, \mathrm{wt}(w)-c p)=d,
\end{gathered}
$$

and it follows as in case 1 that $w$ is well-behaved.

Case 3: The only remaining possibility for the values of $r_{1}$ and $r_{2}$ is $r_{1}=r_{2}=0$. In this case $w$ may be written in the form

$$
w=\left[\left[a_{1}^{p}, q_{2} a_{2}^{p}, \cdots, q_{s} a_{s}^{p},\left(q_{1}-1\right) a_{1}^{p}\right], r_{3} a_{3}, \cdots, r_{s} a_{s}\right],
$$

and so $w \in\left[M_{\left(c^{\prime}\right)}, d^{\prime} G\right]$ with $c^{\prime}=\sum_{j=1}^{s} q_{j}$ and $d^{\prime}=\sum_{j=1}^{s} r_{j}$. This time not only $c^{\prime}=c$ but also

$$
d^{\prime}=\mathrm{wt}(w)-p \sum_{j=1}^{s} q_{j}=\mathrm{wt}(w)-c p=\max (0, \mathrm{wt}(w)-c p)=d .
$$

Anyway, $w \in\left[M_{(c)}, d G\right]$.

This completes the proof of the lemma.

8.8 Lemma. Every non-trivial elementary element of $G^{\prime}$ is well behaved.

Proof. Let $w$ be a non-trivial elementary element of $G^{\prime}$, and let $\delta$ be the degree function of $w$. Let $\operatorname{supp} \delta=\left\{a_{1}, \cdots, a_{s}\right\}$ and as in the proof of 8.7 write $a_{j}=g_{i_{j}}$ and $\delta\left(a_{j}\right)=q_{j} p+r_{j}$, for $j=1, \cdots, s$. Let the subscripting of the $a_{j}$ 's be so arranged that $w$ is expressed in normal form by 


$$
w=\prod_{j=2}^{t}\left[a_{j}, a_{1}, \delta\right]^{e_{j}}
$$

for some $t$ in the range $2 \leqq t \leqq s$. If $t=2$, then $w$ is well-behaved by 8.7 , so assume $t>2$. This assumption implies that $\delta\left(a_{j}\right)<p^{2}$ for each $j \in\{1, \cdots, s\}$ (if $\delta\left(a_{j}\right)=p^{2}$ for some $j$, then there is only one basic pseudo-commutator with degree function $\delta)$, and this in turn means that $\left(g_{2}, g_{1}, \delta^{\left(i_{j}\right)}\right)$ is basic for each $j$. Using this last observation in conjunction with 8.2 it is not hard to see that

$$
\operatorname{mic}(w)=\min _{j}\left(\operatorname{mic}\left(\left[a_{j}, a_{1}, \delta\right]^{e_{j}}\right) \mid j=2, \cdots, t\right)
$$

unless $\sum_{j=2}^{t} e_{j} \equiv 0 \bmod p$ and $\operatorname{comp}\left(g_{2}, g_{1}, \delta^{\left(i_{1}\right)}\right)<\operatorname{comp}\left(g_{2}, g_{1}, \delta^{\left(i_{j}\right)}\right)$ for each $j \in\{2, \cdots, t\}$; that is, unless

(i) $\sum_{j=2}^{t} e_{j} \equiv 0 \bmod p$,

(ii) $r_{1}=0$, and

(iii) $r_{j} \neq 0$ for $j=2, \cdots, t$.

Note that conditions (i) and (iii) imply that $\operatorname{mic}(w)=\sum_{i=1}^{s} q_{j}+1$. Now it is immediate from the definition of weight that

$$
\operatorname{wt}(w)=\operatorname{wt}\left(\left[a_{j}, a_{1}, \delta\right]^{e_{j}}\right)=\sum_{j=1}^{s} \delta\left(a_{j}\right)=p \sum_{j=1}^{s} q_{j}+\sum_{j=1}^{s} r_{j}
$$

for all $j \in\{2, \cdots, t\}$, so, in view of 8.5 and 8.7 , it remains to prove that if (i), (ii) and (iii) are satisfied then $w \in\left[M_{(c)}, d G\right]$, where $c=\sum q_{j}+1$ and $d=\max (0, w t(w)-c p)=\max \left(0, \Sigma r_{j}-p\right)$. This is proved as follows, and it will be noticed that condition (ii) is irrelevant:

The Jacobi identity gives that

$$
\left[a_{j}, a_{1}, \delta\right]=\left[a_{j}, a_{2}, \delta\right]\left[a_{2}, a_{1}, \delta\right]
$$

for each $j \in\{2, \cdots, t\}$, and it therefore follows from condition (i) that

$$
w=\prod_{j=3}^{t}\left[a_{j}, a_{2}, \delta\right]^{e_{j}} .
$$

Condition (iii) allows each factor $\left[a_{j}, a_{2}, \delta\right]$ in this product to be written in the form

$$
\begin{array}{r}
{\left[\left[\left[a_{j}, a_{2}\right], q_{1} a_{1}^{p}, \cdots, q_{s} a_{s}^{p}\right], r_{1} a_{1},\left(r_{2}-1\right) a_{2}, r_{3} a_{3}, \cdots\right.} \\
\left.\cdots r_{j-1} a_{j-1},\left(r_{j}-1\right) a_{j}, r_{j+1} a_{j+1}, \cdots, r_{s} a_{s}\right]
\end{array}
$$

and so it is immediate that $w \in\left[M_{(c)}, d^{\prime} G\right]$, where $d^{\prime}=\Sigma r_{j}-2$. Since $d^{\prime}$ is obviously no greater than $d$, this completes the proof. 


\section{Proof of Lemma $\mathbf{E}$}

Many of the methods employed in this section have their origin in the Ph.D. thesis of R. A. Bryce (Australian National University 1967).

9.1 LemMa (c.f. 4.2 .5 in [3]). Let $w_{1}, \cdots, w_{m} \in G^{\prime}$ and let $W \in \operatorname{id}\left(G^{\prime}\right)$ (i.e. let $W$ be a fully invariant subgroup of $G$ contained in $\left.G^{\prime}\right)$. If $\Pi_{i=1}^{m}\left[w_{i}, v^{i}\right] \in W$ for all $v \in G$, then $\left[w_{m}, v_{m}^{m}, v_{m-1}^{m-1}, \cdots, v_{1}\right] \in W$ for all $v_{1}, \cdots, v_{m} \in G$.

Proof. The proof is by induction on $m$. For $m=1$ there is nothing to prove, so assume the assertion true for $m=k-1>0$ and consider the case $m=k$.

Arbitrarily choose $v_{1}, \cdots, v_{k} \in G$ and $w_{1}, \cdots, w_{k} \in G^{\prime}$ and assume that

$$
\prod_{i=1}^{k}\left[w_{i}, v^{i}\right] \in W \text { for all } v \in G \text {. }
$$

The object is to show that $\left[w_{k}, v_{k}^{k}, \cdots, v_{1}\right] \in W$.

It is a simple exercise in commutator calculus to check that

$$
\left[w_{i},\left(v_{k} \bar{v}\right)^{i}\right]=\left[w_{i}, v_{k}^{i}\right]\left[w_{i}, \bar{v}^{i}\right]\left[w_{i}, v_{k}^{i}, \bar{v}\right]\left[w_{i}, v_{k}^{i}, \bar{v}^{i-1}\right]^{\vec{v}}
$$

for all positive $i$ and all $\bar{v} \in G$. Hence

$$
\prod_{i=1}^{k}\left[w_{i},\left(v_{k} \bar{v}\right)^{i}\right]=\left(\prod_{i=1}^{k}\left[w_{i}, v_{k}^{i}\right]\right)\left(\prod_{i=1}^{k}\left[w_{i}, \bar{v}^{i}\right]\right)\left[\prod_{i=1}^{k}\left[w_{i}, v_{k}^{i}\right], \bar{v}\right]\left(\prod_{i=1}^{k-1}\left[w_{i+1}, v_{k}^{i+1}, \bar{v}^{i}\right]\right)^{\bar{v}}
$$

for all $\bar{v} \in G$, and so it follows from $\left(^{*}\right)$ and the normality of $W$ in $G$ that

$$
\prod_{i=1}^{k-1}\left[\left[w_{i+1}, v_{k}^{i+1}\right], \bar{v}^{i}\right] \in W \text { for all } \bar{v} \in G \text {. }
$$

By the inductive assumption (i.e. the case $m=k-1$ ) this implies that $\left[\left[w_{k-1+1}, v_{k}^{k-1+1}\right], v_{k-1}^{k-1}, \cdots, v_{1}\right] \in W$, which finishes the proof.

9.2 Lemma (c.f. 4.3.1 in [3]). Let $w_{1}, \cdots, w_{p^{2}-1} \in G^{\prime}$ and let $W \in \operatorname{id}\left(G^{\prime}\right)$. If $\Pi_{a=1}^{p^{2}-1}\left[w_{d}, d v\right] \in W$ for all $v \in G$, then for each $d \in\left\{1, \cdots, p^{2}-1\right\}$ there exists a nonnegative integer $e=e(d)$ such that $\left[w_{d}, u_{1}^{p}, \cdots, u_{d^{*}}^{p}, v_{1}, \cdots, v_{e}\right] \in W$ for all $u_{1}, \cdots, u_{d^{*}}, v_{1}, \cdots, v_{e} \in G$, where $d^{*}=$ int. part $(d / p)$.

Proof. First, two simple observations:

(i) If $a_{1}, \cdots, a_{m} \in G^{\prime}$ and $b \in G$ then it is easy to check by induction on $m$ that

$$
\prod_{k=1}^{m}\left[a_{k}, k b\right]=\prod_{k=1}^{m}\left[a_{k}^{\prime}, b^{k}\right]
$$

where, for $k=1, \cdots, m$,

$$
a_{k}^{\prime}=\prod_{j=k}^{m} a_{j}^{(-1)^{j-k}\left(\begin{array}{l}
j \\
k
\end{array}\right),}
$$

and it is to be noted that $a_{m}^{\prime}=a_{m}$. 
(ii) Because $\left[x, y, z^{p^{2}}\right]$ is a law in $G$ it is easy to see that if for some $w \in G^{\prime}$ and some positive integer $k$ with $1 \leqq k \leqq p^{2}-1$ it is true that $\left[w, v^{k}\right] \in W$ for all $v \in G$, then it is also true that $\left[w, \bar{v}^{p^{h}}\right] \in W$ for all $\bar{v} \in G$ where $p^{h}$ is the highest power of $p$ which divides $k$.

Now to the proof proper. The premise is that

$$
\prod_{d=1}^{p^{2}-1}\left[w_{d}, d v\right] \in W \text { for all } v \in G .
$$

By 9.1 and (i), (ii) above it is clear that $\left(^{*}\right)$ implies that

$$
\left[w_{p^{2}-1}, \bar{v}_{p^{2}-1}, \cdots, \bar{v}_{p^{2}-p+1}, \bar{v}_{p^{2}-p}^{p}, \bar{v}_{p^{2}-p-1}, \cdots, \bar{v}_{p^{2}-2 p+1}, \bar{v}_{p^{2}-2 p}^{p}, \cdots, \text { etc } \cdots, \bar{v}_{1}\right] \in W
$$

for all $\bar{v}_{1}, \cdots, \bar{v}_{p^{2}-1} \in G$. Thus $\left(^{*}\right)$ implies that

$$
\left[w_{p^{2}-1}, u_{1}^{p}, \cdots, u_{p-1}^{p}, v_{1}, \cdots, v_{p^{2}-p}\right] \in W \text { for all } u_{1}, \cdots, u_{p-1}, v_{1}, \cdots, v_{p^{2}-p} \in G,
$$

and since int. part $\left(\left(p^{2}-1\right) / p\right)=p-1$ this establishes the case $d=p^{2}-1$. The remaining cases are handled by induction as follows:

Let $r \in\left\{1, \cdots, p^{2}-2\right\}$ and assume inductively that for each $d$ in the range $r<d<p^{2}$ there exists an $e=e(d)$ such that

$$
\left[w_{d}, u_{1}^{p}, \cdots, u_{d^{*}}^{p}, v_{1}, \cdots, v_{e}\right] \in W \text { for all } u_{1}, \cdots, u_{d^{*}}, v_{1}, \cdots, v_{e} \in G .
$$

Since

$$
\left[w_{d}, d v\right]=\left[w_{d},\left(d^{*} p+\left(d-d^{*} p\right)\right) v\right]=\left[w_{d}, d^{*} v^{p},\left(d-d^{*} p\right) v\right],
$$

this inductive assumption implies that there exists a non-negative integer $l$ such that if $r<d<p^{2}$ then

$$
\left[w_{d}, d v, v_{1}, \cdots, v_{l}\right] \in W \text { for all } v_{1}, \cdots, v_{l}, v \in G .
$$

From $\left({ }^{*}\right)$ and $(* *)$ it follows that $\Pi_{d=1}^{r}\left[\left[w_{d}, v_{1}, \cdots, v_{l}\right], d v\right] \in W$ for all $v_{1}, \cdots, v_{l}, v \in G$, and, again by 9.1 and (i), (ii) above, this in turn implies that

$$
\left[\left[w_{r}, v_{1}, \cdots, v_{l}\right], \vec{v}_{r}, \cdots, \bar{v}_{r^{*} p+1}, \bar{v}_{r^{*} p}^{p}, \vec{v}_{r^{*} p-1}, \cdots \text { etc } \cdots, \bar{v}_{1}\right] \in W
$$

for all $v_{1}, \cdots, v_{l}, \bar{v}_{1}, \cdots, \bar{v}_{\mathrm{r}} \in G$. The case $d=r$ follows, and the induction is complete.

9.3 Lemma (c.f. 4.3.2 in [3]). Let $W \in \operatorname{id}\left(G^{\prime}\right)$ and let $\left\{a_{1}, \cdots, a_{s}\right\} \subseteq$ g. Let $\Phi$ be the set of all mappings from $\{1, \cdots, s\}$ into $\left\{0,1, \cdots, p^{2}-1\right\}$ and for each $\phi \in \Phi$ let $w_{\phi} \in G^{\prime} \cap \operatorname{gp}\left(\mathfrak{g} \backslash\left\{a_{1}, \cdots, a_{s}\right\}\right)$. If

$$
w=\prod_{\phi \in \Phi}\left[w_{\phi}, 1 \phi a_{1}, \cdots, s \phi a_{s}\right] \in W,
$$

then for each $\phi \in \Phi$ there exists a non-negative integer $e=e(\phi)$ such that $\left[w_{\phi}, u_{1}^{p}, \cdots, u_{\phi *}^{p}, v_{1}, \cdots, v_{e}\right] \in W$ for all $u_{1}, \cdots, u_{\phi^{*}}, v_{1}, \cdots, v_{e} \in G$, where $\phi^{*}=$ $\sum_{i=1}^{s}$ int. part $\left.i \phi / p\right)$. 
ProOF. For $s=1$ the premise reduces to $\prod_{d=0}^{p^{2}-1}\left[w_{d}, d a_{1}\right] \in W$ where $a_{1}$ is a free generator of $G$ not "involved" in any $w_{d}$. Since for any $v \in G$ (including $v=1)$ there exists an endomorphism $\alpha$ of $G$ such that $a_{1} \alpha=v$ and $w_{d} \alpha=w_{d}$ for each $d$, this is equivalent to saying that $w_{0} \in W$ and $\prod_{d=1}^{p^{2}-1}\left[w_{d}, d v\right] \in W$ for all $v \in G$. At this point 9.2 applies, and the case $s=1$ of the lemma is established.

Now suppose $s>1$. For each $d \in\left\{0, \cdots, p^{2}-1\right\}$ set

$$
w_{d}=\prod_{\phi \in \Phi_{d}}\left[w_{\phi}, 1 \phi a_{1}, \cdots,(s-1) \phi a_{s-1}\right],
$$

where $\Phi_{d}=\{\phi \in \Phi \mid s \phi=d\}$. Then, of course, $\Pi_{d=0}^{p^{2}-1}\left[w_{d}, d a_{s}\right]=w \in W$, and as with the case $s=1$ this implies that for each $d=\left\{0, \cdots, p^{2}-1\right\}$ there exists a non-negative integer $e=e(d)$ such that $\left[w_{d}, u_{1}^{p}, \cdots, u_{d *}^{p}, v_{1}, \cdots, v_{e}\right] \in W$ for all $u_{1}, \cdots, u_{d^{*}}, v_{1}, \cdots, v_{e} \in G$. Choose $u_{1}, \cdots, u_{d^{*}}, v_{1}, \cdots, v_{e}$ arbitrarily and for each $\phi \in \Phi_{d}$ set $\bar{w}_{\phi}=\left[w_{\phi}, u_{1}^{p}, \cdots, u_{d^{*}}^{p}, v_{1}, \cdots, v_{e}\right]$. Then

$$
\prod_{\phi \in \Phi_{d}}\left[\bar{w}_{\phi}, 1 \phi a_{1}, \cdots,(s-1) \phi a_{s-1}\right]=\left[w_{d}, u_{1}^{p}, \cdots, u_{d^{*}}^{p}, v_{1}, \cdots, v_{e}\right] \in W,
$$

and the obvious inductive step completes the proof.

Now consider Lemma E (4.6). An arbitrary non-trivial special element $w$ of $G^{\prime}$ can be expressed in normal form as

$$
w=\prod_{j=1}^{t}\left[g_{2}, g_{1}, \delta_{j}\right]^{e_{j}},
$$

where, of course $e_{j} \neq 0 \bmod p$ and $\delta_{j}\left(g_{1}\right)=\delta_{j}\left(g_{2}\right)=1$ for each $j$. Suppose that $\bigcup_{j=1}^{t} \operatorname{supp} \delta_{j}=\left\{g_{1}, g_{2}, a_{1}, \cdots, a_{s}\right\}$. If $s=0$, then $w=\left[g_{2}, g_{1}\right]^{e_{1}}$ and in that case Lemma $\mathrm{E}$ (4.6) reduces to a triviality. so assume $s>0$.

Let $\Phi$ be as in 9.3 and for each $\phi \in \Phi$ set

Then

$$
w_{\phi}= \begin{cases}{\left[g_{2}, g_{1}\right]^{e_{j}}} & \text { if } i \phi=\delta_{j}\left(a_{i}\right) \text { for } i=1, \cdots, s, \\ 1 & \text { otherwise. }\end{cases}
$$

$$
w=\prod_{\phi \in \Phi}\left[w_{\phi}, 1 \phi a_{1}, \cdots, s \phi a_{s}\right],
$$

and so 9.3 gives that for each $\phi \in \Phi$ there exists a non-negative integer $e=e(\phi)$ such that, if $W$ is the fully invariant closure of $w$, then $\left[w_{\phi}, u_{1}^{p}, \cdots, u_{\phi^{*}}^{p}, v_{1}, \cdots, v_{e}\right] \in W$ for all $u_{1}, \cdots, u_{p^{*}}, v_{1}, \cdots, v_{e} \in G$. Now by definition

$$
\begin{aligned}
c & =\operatorname{comp}(w) \\
& =\min _{j}\left(1+\sum_{i=1}^{s} \text { int. } \operatorname{part}\left(\delta_{j}\left(a_{i}\right) / p\right) \mid j=1, \cdots, t\right) \\
& =\min _{\phi}\left(\phi^{*} \mid w_{\phi} \neq 1\right)+1,
\end{aligned}
$$


and consequently there exists a $\phi \in \Phi$ and a non-negative integer $e$ such that $w_{\phi}=\left[g_{2}, g_{1}\right]^{e_{j}} \neq 1$ and

$$
\left[w_{\phi}, u_{2}^{p}, \cdots, u_{c}^{p}, v_{1}, \cdots, v_{e}\right] \in W \text { for all } u_{2}, \cdots, u_{c}, v_{1}, \cdots, v_{e} \in G .
$$

Clearly, in the notation of 6.1 , this means that $W \geqq V(c, e)$. So for the proof of Lemma $\mathrm{E}$ (4.6) it now only remains to show that $V(c, e) \geqq\left[M_{(c)}, e^{\prime} G\right]$ for some $e^{\prime}$. In fact, though:

9.4 Lemma. For all $c \geqq 1$ and all $e \geqq 0, V(c, e) \geqq\left[M_{(c)},(e+1) G\right]$.

Proof. In view of 6.2 it is only required to show that $V(c, e) \geqq U(c, e+1)$, and for this it is sufficient to prove that

$$
\left[u_{1}^{p}, \cdots, u_{c}^{p}, v_{1}, \cdots, v_{e+1}\right] \in V(c, e)
$$

for all $u_{1}, \cdots, u_{c}, v_{1}, \cdots, v_{e+1} \in G$. But, by the Jacobi identity,

$$
\left[u_{1}^{p}, u_{2}^{p}, v_{e+1}\right]=\left[u_{1}^{p}, v_{e+1}, u_{2}^{p}\right]\left[v_{e+1}, u_{2}^{p}, u_{1}^{p}\right],
$$

and the result follows.

\section{Proof of Lemma F}

The proof of Lemma $F(4.7)$ depends on the characterisation of $G^{p^{2}} \cap G^{\prime}$ given by the lemma below. The idea for the proof of this lemma was suggested to me by L. G. Kovács.

10.1 LeMma. If $V$ denotes the fully invariant closure of $g_{2}^{-p^{2}} g_{1}^{-p^{2}}\left(g_{1} g_{2}\right)^{p^{2}}$ in $G$, then $V=G^{p^{2}} \cap G^{\prime}$.

Proof. Since $\left(g_{1} g_{2}\right)^{p^{2}}=g_{1}^{p^{2}} g_{2}^{p^{2}} c$ for some $c \in G^{\prime}$, it is clear that $V \leqq G^{p^{2}} \cap G^{\prime}$. Hence if $H$ denotes the relatively free group $G / V$, then it is sufficient to prove that $H^{p^{2}} \cap H^{\prime}=\{1\}$.

Let $w \in H^{p^{2}}$. From the definition of $H$ it is clear that $(a b)^{p^{2}}=a^{p^{2}} b^{p^{2}}=b^{p^{2}} a^{p^{2}}$ for all $a, b \in H$, (the second equality holds because $\left[x^{p^{2}}, y^{p^{2}}\right]$ is a law in $G$ ) and it follows that $w$ can be expressed in the form

$$
w=a_{1}^{\alpha_{1} p^{2}} \cdots a_{k}^{\alpha_{k} p^{2}}
$$

where $a_{1}, \cdots, a_{k}$ are pairwise distinct members of some free generating set $\mathfrak{H}$ for $H$ and $\alpha_{1}, \cdots, \alpha_{k}$ are integers. Now assume additionally that $w \in H^{\prime}$. If for $j=1, \cdots, k$ endomorphisms $\sigma_{j}$ of $H$ are defined by $a_{j} \sigma_{j}=a_{j}$ and $h \sigma_{j}=1$ for all $h \in \mathfrak{S} \backslash\left\{a_{j}\right\}$, then it follows that $a_{j}^{\alpha_{j} p^{2}}=w \sigma_{j} \in H^{\prime}$ for each $j$. But, of course, $g p(h) \cap H^{\prime}=\{1\}$ for all $h \in \mathfrak{G}$ and therefore $a_{1}^{\alpha_{1} p^{2}}=\cdots=a_{k}^{\alpha_{k} p^{2}}=1$, which means that $w=1$. The lemma is proved.

In view of 10.1 it is sufficient for the proof of Lemma $F$ (4.7) to show that $g_{2}^{-p^{2}} g_{1}^{-p^{2}}\left(g_{1} g_{2}\right)^{p^{2}} \in M_{(p)}$, or equivalently that $\left(g_{1} g_{2}\right)^{p^{2}} \equiv g_{1}^{p^{2}} g_{2}^{p^{2}} \bmod M_{(p)}$. To do 
this, first write $\left(g_{1} g_{2}\right)^{p}=g_{1}^{p} g_{2}^{p} d$, (where, of course, $d \in G^{\prime}$ ) and note that $g_{1}^{p}, g_{2}^{p}, d \in M$. Now $M / M_{(p)}$ is a $p$-group of class less than $p$ and as such is regular. Thus

$$
\left(g_{1} g_{2}\right)^{p^{2}}=\left(g_{1}^{p} g_{2}^{p} d\right)^{p} \equiv\left(g_{1}^{p}\right)^{p}\left(g_{2}^{p}\right)^{p} d^{p} \bmod M_{(p)},
$$

and the result follows since $d^{p}=1 .\left(G^{\prime}\right.$ has exponent $p$.)

\title{
References
}

[1] Warren Brisely, 'On varieties of metabelian p-groups and their laws', J. Austral. Math. Soc. 7 (1967), 64-80.

[2] M. S. Brooks, 'On lattices of varieties of metabelian groups', J. Austral. Math. Soc. 12 (1971), 161-166.

[3] R. A. Bryce, 'Metabelian groups and varieties', Philos. Trans. Roy. Soc. London, Ser. A. 266 (1970) 281-355.

[4] D. E. Cohen, 'On the laws of a metabelian variety', J. Algebra 5 (1967), 267-273.

[5] L. G. Kovács, 'The descending chain condition in join-continuous modular lattices', $J$. Austral. Math. Soc. 10 (1969), 1-4.

[6] L. G. Kovács and M. F. Newman, 'On varieties of metabelian groups', in preparation.

[7] L. G. Kovács and M. F. Newman, 'On non-Cross varieties of groups', J. Austral. Math. Soc. 12 (1971), 129-144.

[8] Hans Liebeck, 'Concerning nilpotent wreath products', Proc. Camb. Philos. Soc. 58 (1962), $443-451$.

[9] R. C. Lyndon, 'Two notes on nilpotent groups', Proc. Amer. Math. Soc. 3 (1952), 579-583.

[10] H. Meier-Wunderli, 'Metabelsche Gruppen', Comment. Math. Helvet. 25 (1951), 1-10.

[11] Hanna Neumann, Varieties of groups (Springer-Verlag, 1967).

[12] T. G. Newman, 'The descending chain condition in modular lattices', J. Austral. Math. Soc. (to appear).

[13] Paul M. Weichsel, ‘On metabelian p-groups', J. Austral. Math. Soc. 7 (1967), 55-63.

\author{
Australian National University \\ Present address: \\ Department of Mathematics \\ Teeside Polytechnic \\ England
}

